\title{
Checklist of Sphagnum-dwelling testate amoebae in Bulgaria
}

\author{
Nikola Bankov, Milcho Todorov $\ddagger$, Anna Ganeva ${ }^{\ddagger}$ \\ ‡ Institute of Biodiversity and Ecosystem Research, Bulgarian Academy of Sciences, Sofia, Bulgaria
}

Corresponding author: Milcho Todorov (todorovmilcho@gmail.com)

Academic editor: Anna Maria Fiore-Donno

Received: 26 Mar 2018| Accepted: 16 May 2018| Published: 22 May 2018

Citation: Bankov N, Todorov M, Ganeva A (2018) Checklist of Sphagnum-dwelling testate amoebae in Bulgaria.

Biodiversity Data Journal 6: e25295. https://doi.org/10.3897/BDJ.6.e25295

ZooBank: urn:Isid:zoobank.org:pub:D9C64849-0CBD-48D7-96B1-F43E5631875D

\begin{abstract}
\section{Background}

Until now, a complete checklist of Sphagnum-dwelling testate amoebae in Bulgaria has never been published. Records for species diversity and distribution in the country were scattered in many faunistic and ecological publications. The aim of the present study is to summarise all data for the species distribution at the level of country by reviewing the existing literature and by additional data obtained in our research over the past two years.
\end{abstract}

\section{New information}

The checklist comprises 171 species, classified into 43 genera, 20 families, three orders, three classes and three phyla. We present data for 16 new Sphagnum-dwelling testate amoebae in Bulgaria and new distribution data for 134 species. Of them, 99 species are recorded from Stara Planina Mt., for which there was no available data to date. Additionally are recorded 69 new species for Pirin Mt., 21 for Vitosha Mt. and 18 for Rila Mt. Thirty six species are synonymised according to the latest taxonomic changes. Two misidentified taxa (Euglypha brachiata Penard, 1902 and Difflugia compressa var. africana GauthierLièvre et Thomas, 1958) are transferred into valid species E. acanthophora and Zivkovicia 
compressa, respectively. Three of the recorded species have not been included in the checklist, because they are currently not refering to testate amoebae (Cochliopodium bilimbosum (Auerbach 1856) and Cochliopodium echinatum Korotneef, 1879 are gymnamoebae (naked amoebae) and Microgromia elegantula (Penard 1904) = Paralieberkuehnia elegantula (Penard 1904) is freshwater foraminifera).

\section{Keywords}

Amoebozoa, Cercozoa, Stramenopiles, biodiversity, distribution, Sphagnum mosses, Bulgaria

\section{Introduction}

Testate amoebae are a polyphyletic assemblage of at least three major, unrelated taxonomic groups of eukaryotes (Adl SM et al. 2012). They are present in most terrestrial and freshwater environments and more marginally in brackish and marine habitats. They are especially abundant and diverse in Sphagnum mosses. Research on testate amoebae have increased significantly over the past two decades due to their increasing use in different applied aspects: as a bioindicators for palaeoecological studies, in environmental monitoring, pollution hazards, ecotoxicology, studies on their role in the cycling of elements in terrestrial ecosystems, biogeographical and evolutionary studiesetc. (Aoki, Yoshiyuki et al. 2007, Booth 2002, Charman 2001, Charman et al. 1998, Lamentowicz and Mitchell 2005, Mitchell et al. 2008, Nguyen-Viet et al. 2007, Swindles et al. 2008, Swindles et al. 2015, Qin et al. 2013).

Studies on Sphagnum-dwelling testate amoebae in Bulgaria started at the beginning of the 20th century, when Pateff (1924), Pateff (1928) published his works on freshwater Rhizopoda in the country. He recorded a total of 118 rhizopods, of which 52 testate amoebae were from Sphagnum mosses in the Rila, Rhodopes and Vitosha Mountains. Later, Valkanov (1932), Valkanov (1934) published results of his studies on the fauna of alpine lakes in the Rila and Pirin Mountains and recorded many testate amoebae, which are typical inhabitants of peat mosses. Unfortunately, they were recorded along with all other species and no distinction was made amongst the species found in the lakes themselves and in the Sphagnum mosses from the lake shore. In the 60s and 70s of the 20th century, several publications on testate amoebae from the Vitosha, Rhodopes and Pirin Mountains were published (Golemansky 1965, Golemansky 1966, Golemansky 1968, Golemansky 1974), including a lot of data on Sphagnum-dwelling testate amoebae. At the end of the 20th and beginning of the 21th century, the number of publications concerning various aspects of taxonomy, systematics, morphology and ecology of sphagnicolous testate amoebae in Bulgaria increased considerably (Golemansky and Todorov 1990, Golemansky and Todorov 1993, Golemansky and Todorov 2006, Golemansky and Todorov 1985, Golemansky et al. 2006, Heger et al. 2010, Kosakyan et al. 2012, Todorov 1993, Todorov 2004, Todorov 2005, Todorov 2010, Todorov and Golemansky 1995, Todorov and 
Golemansky 2000, Todorov et al. 2010, Todorov et al. 2009). All data in the abovementioned publications have never been summarised in one place and that is the reason why the main goal of the present paper is to unite this scattered information and, together with the new data obtained from our research over the past two years, to present a checklist of all known to date Sphagnum-dwelling testate amoebae in Bulgaria. Data on the distribution, habitats, regions, localities, altitude, authors and sources of information are provided in Suppl. material 1. Some typical and a few rare sphagnicolous testate amoebae, recorded from the country, are illustrated in Figs 1, 2, 3, 4.

\section{Materials and methods}

The data presented in the checklist are based primarily on published information concerning Sphagnum-dwelling testate amoebae in Bulgaria. In addition, data from our research conducted over the past two years are also included. We have tried to explore the diversity and distribution of testate amoebae in the main areas of the country where peat mosses are more widespread. Genus Sphagnum in Bulgaria numbers 27 species (Natcheva and Ganeva 2005) distributed in mires in mountain areas of the country above the tree belt or in mires in coniferous forest, these being an important component of habitat 91D0 (Bog Woodland) of Directive 92/43/EEC. Some of the species (e.g. Sphagnum capillifolium (Ehrh.) Hedw., S. subsecundum Nees, S. girgensohnii Russ., S. centrale C.E.O.Jensen, S. platyphyllum (Lindb. ex. Braithw.) Sull. ex Warnst., S. squarrosum Crome, S. teres (Schimp.) Ängstr.) are more widespread in mires of Western Stara Planina, Rhodopes, Vitosha, Rila and Pirin Mountains, where, with other bryophytes and sedges, they comprise the specific mire flora.

The material for the present study was extracted from wet Sphagnum mosses, gathered at Vitosha and Western Stara Planina Mountains in 2016 and Rila and Pirin Mountains in 2017. A total of 109 samples from 18 localities were sampled and examined. All data, concerning sampling localities including date, region, altitude, coordinates, Sphagnum moss species, as well as many physical and chemical parameters of the sampling sites, are provided in Suppl. material 2.

Testate amoebae were extracted from fresh Sphagnum mosses at the sampling site and concentrated by sieving $(350 \mu \mathrm{m})$. The resulting fraction $(50 \mathrm{ml})$ was observed with an optical microscope "Amplival" (Zeiss-Jena) using 40x objective and 10x ocular lens. For scanning electron microscopy (SEM), specimens were isolated by searching through small isolates of material in a petri dish. Specimens were extracted using a glass micropipette, washed several times in distilled water and then individual shells were positioned with a single-hair brush on a previously mounted double-sided adhesive tape on a standard aluminium stub and air-dried. The shells were coated evenly with gold in a vacuum coating unit. The photomicrographs were obtained using a JEOL JSM-5510, operating at $10 \mathrm{kV}$. 


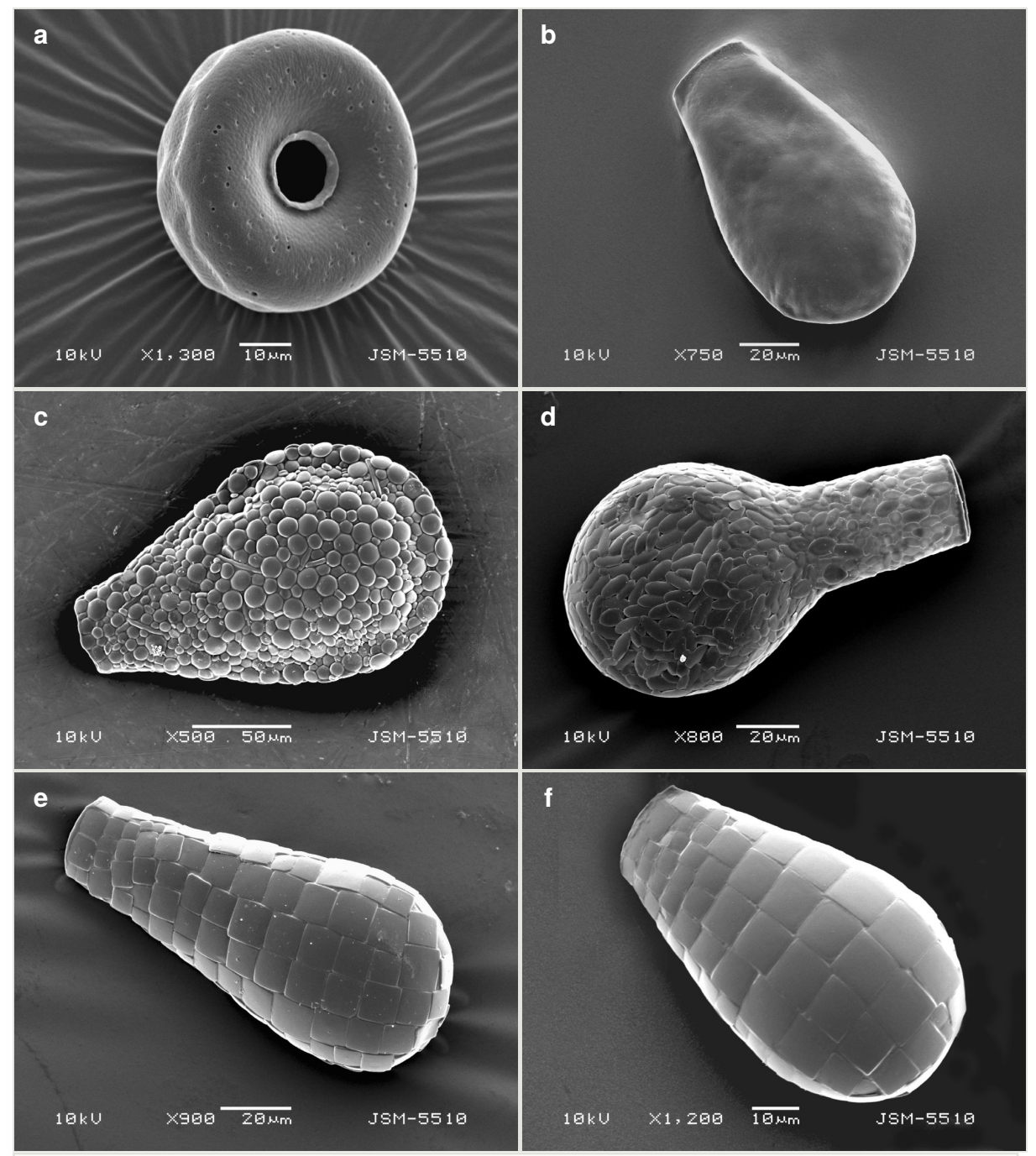

Figure 1.

Characteristic Sphagnum-dwelling testate amoebae from the order Arcellinida.

a: Arcella intermedia (Deflandre) (Arcellidae) doi

b: Hyalosphenia papilio (Leidy) (Hyalospheniidae) doi

c: Gibbocarina galeata (Penard) (Hyalospheniidae) doi

d: Padaungiella lageniformis (Penard) (Hyalospheniidae) doi

e: Quadrulella longicollis Taranek (Hyalospheniidae) doi

f: Quadrulella symmetrica (Wallich) (Hyalospheniidae) doi 


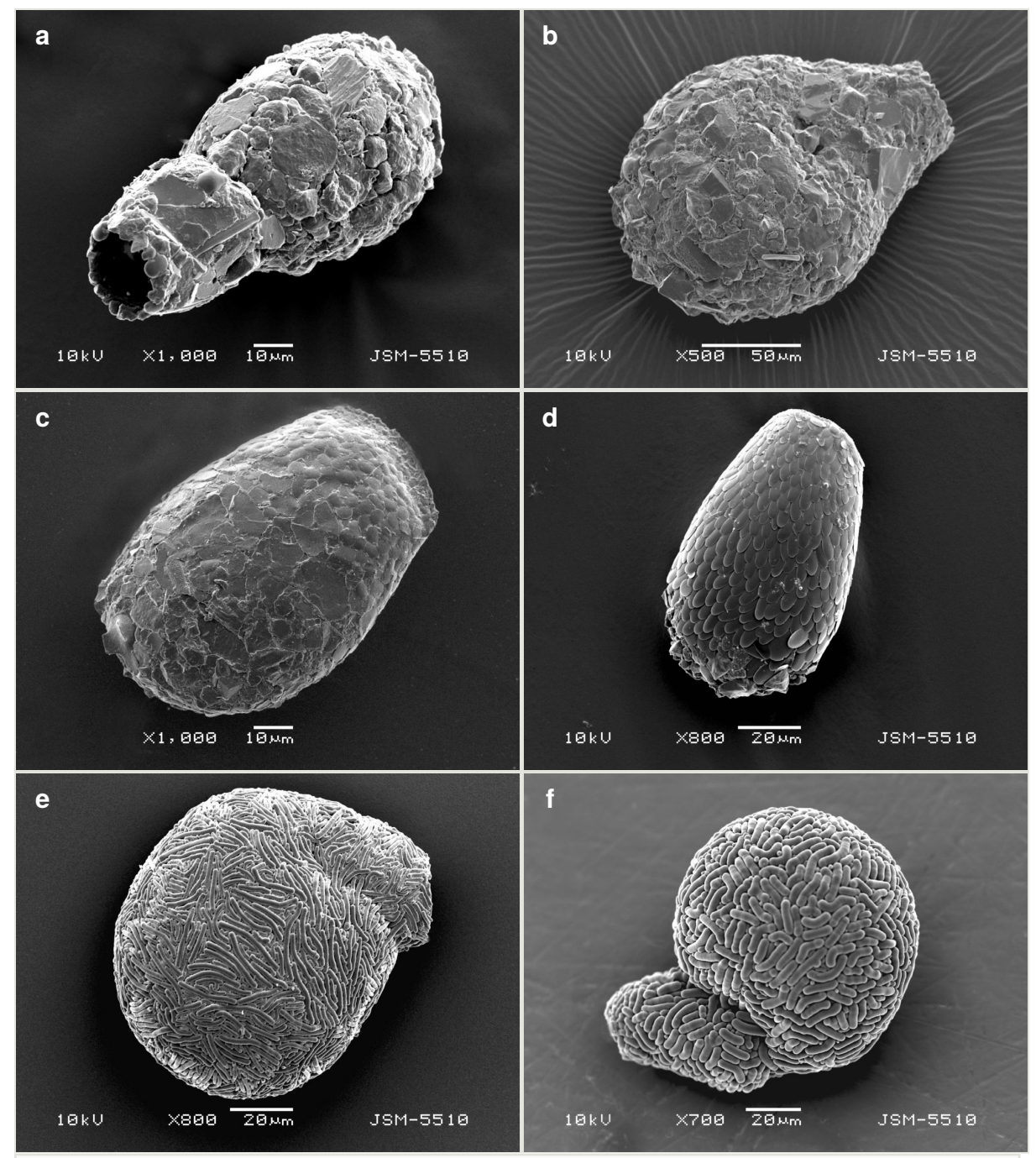

Figure 2.

Characteristic Sphagnum-dwelling testate amoebae from the order Arcellinida.

a: Lagenodifflugia bryophila (Penard) (Difflugiidae) doi

b: Zivkovicia compressa (Carter) (Difflugiidae) doi

c: Heleopera rosea Penard (Incertae sedis Arcellinida) doi

d: Heleopera petricola Leidy (Incertae sedis Arcellinida) doi

e: Lesquereusia spiralis (Ehrenberg) (Incertae sedis Arcellinida) doi

f: Lesquereusia epistomium Penard (Incertae sedis Arcellinida) doi 


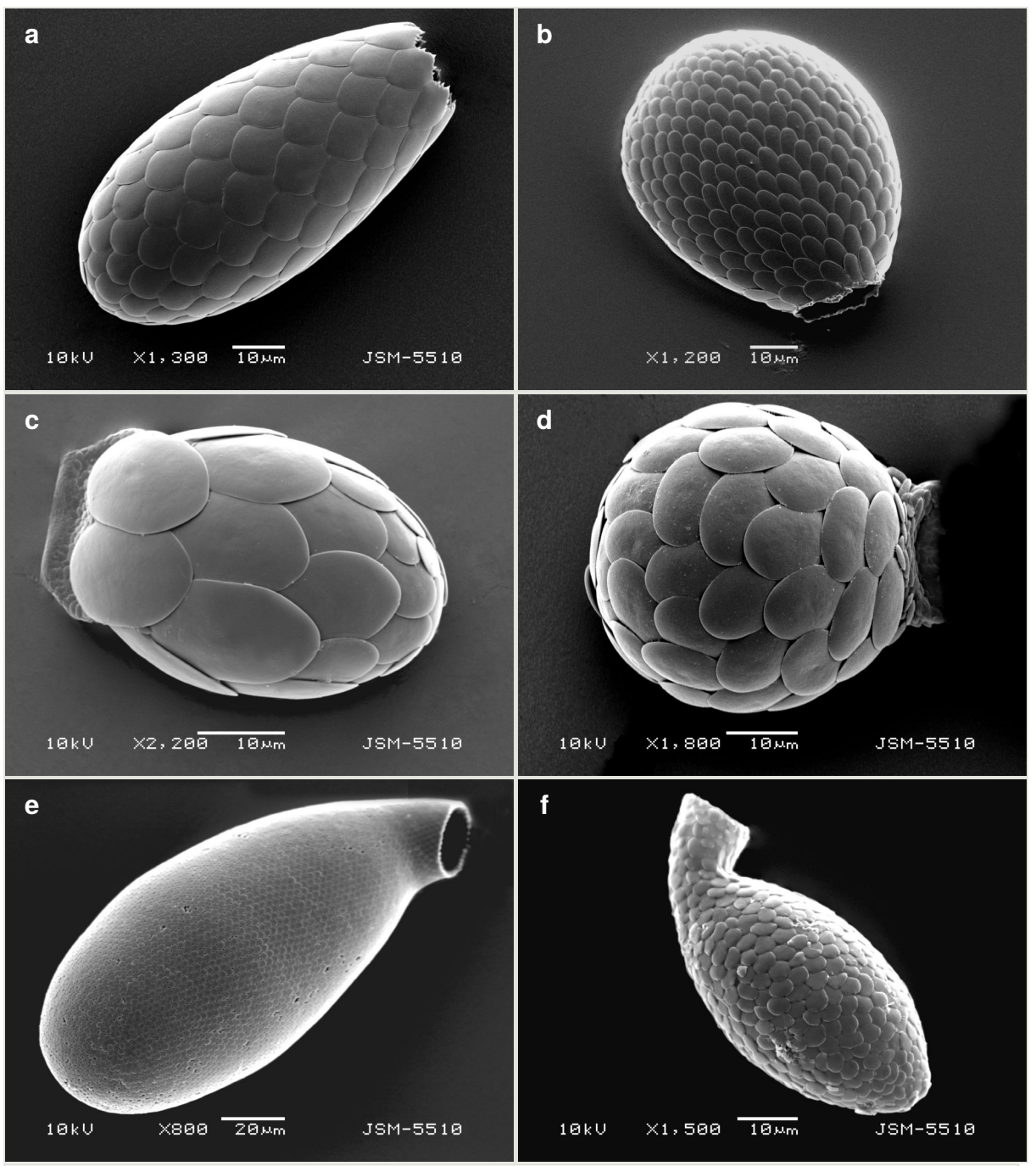

Figure 3.

Characteristic Sphagnum-dwelling testate amoebae from the order Euglyphida.
a: Euglypha tuberculata Dujardin (Euglyphidae) doi
b: Assulina seminulum (Ehrenberg) (Assulinidae) doi
c: Sphenoderia fissirostris Penard (Sphenoderiidae) doi
d: Sphenoderia lenta Schlumberger (Sphenoderiidae) doi
e: Cyphoderia major (Penard) (Cyphoderiidae) doi
f: Campascus minutus Penard (Cyphoderiidae) doi 

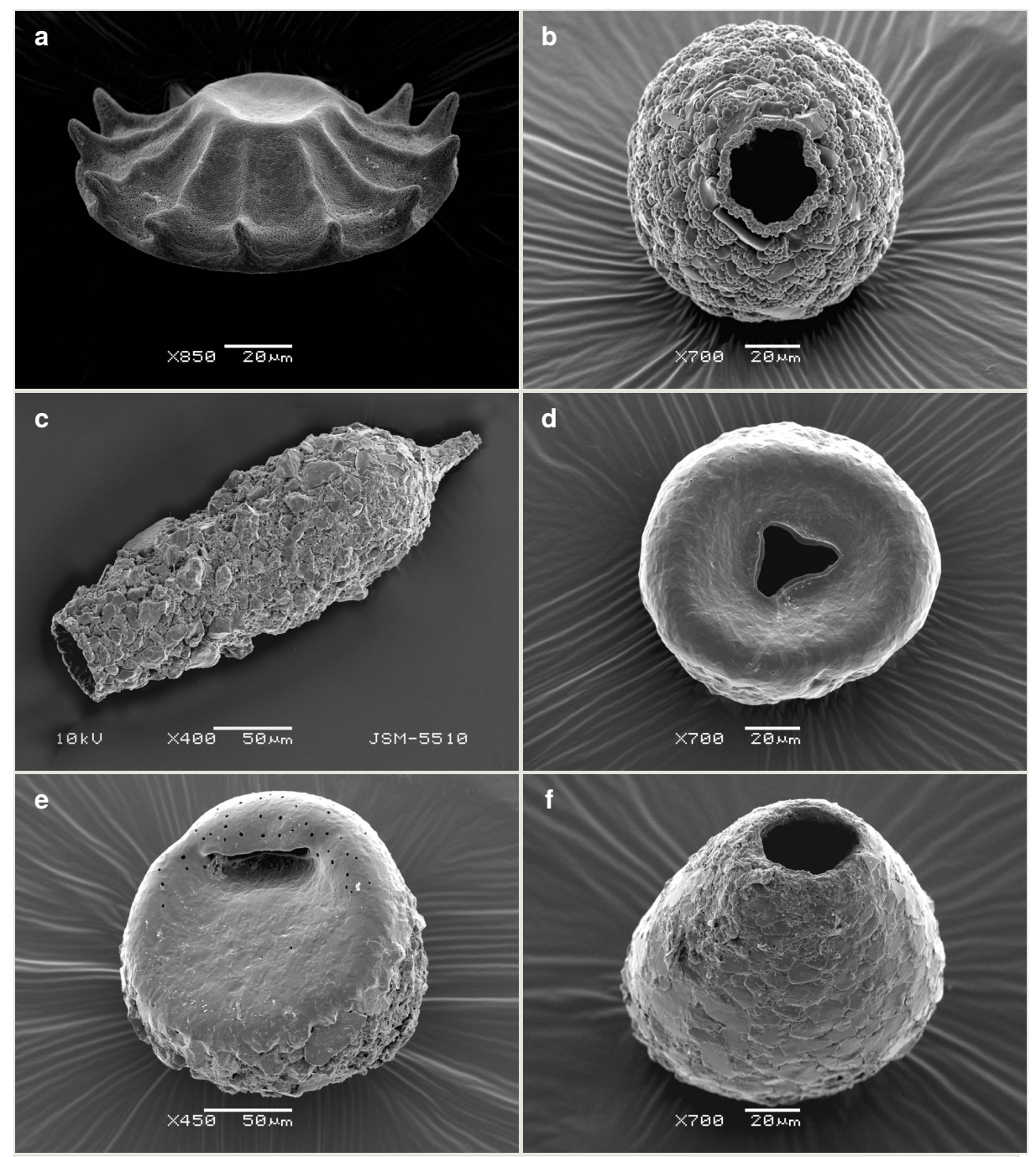

Figure 4.

Rare or incidentally occurring testate amoebae in Sphagum mosses.
a: Arcella dentata Ehrenberg (Arcellidae) doi
b: Netzelia tuberculata (Wallich) (Netzeliidae) doi
c: Difflugia acuminata Ehrenberg (Difflugiidae) doi
d: Trigonopyxis arcula (Leidy) (Trigonopyxidae) doi
e: Bullinularia indica (Penard) (Plagiopyxidae) doi
f: Awerintzewia cyclostoma (Penard) (Incertae sedis Arcellinida) doi

The checklist is annotated with information regarding the synonymous names (if available), species distribution in Sphagnum mosses in Bulgaria, the relevant literature sources and notes (mainly taxonomic). The higher classification used here follows Adl SM et al. 2012 and Meisterfeld (2002a), Meisterfeld (2002b). Taxonomic revision of some genera and 
families, as well as numerous taxonomic and nomenclature changes, based on recent molecular studies and subsequent phylogenetic reconstructions, have also been taken into account (Blandenier et al. 2017, Chatelain et al. 2013, Gomaa et al. 2013, Gomaa et al. 2017, Heger et al. 2010, Kosakyan et al. 2016a, Kosakyan et al. 2012, Kosakyan et al. 2016b, Lahr et al. 2013, Lara et al. 2007, Mazei and Warren 2012, Mazei and Warren 2014, Mazei and Warren 2015).

The list does not include separate varieties and forms recorded from Bulgaria, despite the fact that, according to the International Code of Zoological Nomenclature, article 45.6.3, when the name was published before 1961 using the abbreviation 'var.' or 'f.', it is deemed to be subspecific rather than infrasubspecific. However, due to the fact that many of these taxa have not sufficiently detailed descriptions, which in many cases are based on signs that do not have much taxonomic significance (small differences in size, the presence or absence of spines/horns and their number, the number of lobes of the aperture etc.), these taxa remain with unclear taxonomic status. So, until the clarifying of their status with the help of combined morphological and molecular approaches and full confirmation of their validity, we prefere to adopt a conservative position and consider these taxa as the product of the phenotipic plasticity of nominal species. Nevertheless, in 'Notes' to each nominal species, we have included all the records for these infrasubspecific taxa, in the event that some of them may be raised in rank in the future.

\section{Checklist of Sphagnum-dwelling testate amoebae in Bulgaria}

\section{Kingdom Protozoa Owen, 1858}

Subkingdom Neozoa Cavalier-Smith, 1993

Phylum Amoebozoa Lühe, 1913, emend. Cavalier-Smith, 1998

Subphylum Lobosa Carpenter, 1861, emend. Cavalier-Smith, 2009

Class Tubulinea Smirnov et al., 2005

Order Arcellinida Kent, 1880

Suborder Arcellina Haeckel, 1894

Family Microcoryciidae de Saedeler, 1934

Genus Microcorycia Cockerell, 1911 
Microcorycia flava (Greeff, 1866), emend. Penard, 1902

Nomenclature:

Amphizonella flava Greeff, 1866

Corycia flava Greeff, 1866

Corycia Dujardini? Gagliardi, 1871 (in part)

Distribution: Rhodopes Mt. (Golemansky et al. 2006).

Family Microchlamyidae Ogden, 1985

Genus Microchlamys Cockerell, 1911

Microchlamys patella (Claparede and Lachmann, 1859) Cockerell, 1911

Nomenclature:

Pseudochlamys patella Claparede and Lachmann, 1859

Distribution: Pirin Mt. (new data); Rhodopes Mt. (Golemansky et al. 2006); Rila Mt. (Golemansky and Todorov 1993, Todorov and Golemansky 2000, Todorov 2004, Todorov 2005, new data); Stara Planina Mt. (new data); Vitosha Mt. (new data).

Family Arcellidae Ehrenberg, 1843

Genus Arcella Ehrenberg, 1832

Arcella arenaria Greeff, 1866

Nomenclature:

Arcella aureola Maggi, 1883

Arcella microstoma Penard, 1890

Distribution: Pirin Mt. (new data); Rhodopes Mt. (Golemansky et al. 2006); Rila Mt. (Golemansky and Todorov 1993, Todorov and Golemansky 2000, new data); Vitosha Mt. (Golemansky 1965, Golemansky and Todorov 1990, new data).

Arcella bathystoma Deflandre, 1928

Distribution: Rila Mt. (new data). 


\section{Arcella catinus Penard, 1890}

Nomenclature:

Arcella vulgaris Leidy, 1879 (in part)

Arcella artocrea Penard, 1902

Arcella vulgaris var. compressa Cash, 1905

Arcella catinus var. australis Playfair, 1918

Distribution: Pirin Mt. (new data); Rhodopes Mt. (Golemansky 1968, Golemansky et al. 2006); Rila Mt. (Golemansky and Todorov 1993, new data); Stara Planina Mt. (new data); Vitosha Mt. (Pateff 1928, Golemansky 1965, Golemansky and Todorov 1990, Todorov 1993, Todorov and Golemansky 1995, new data).

\section{Arcella dentata Ehrenberg, 1830}

Nomenclature:

Arcella stellaris Perty, 1852

Arcella Okeni Perty, 1852

Arcella stellata Ehrenberg, 1854

Distribution: Pirin Mt. (new data); Rhodopes Mt. (Pateff 1924, Golemansky et al. 2006).

\section{Arcella discoides Ehrenberg, 1871}

Nomenclature:

Arcella discoidea Ehrenberg, 1843

Distribution: Pirin Mt. (Golemansky 1974, new data); Rhodopes Mt. (Pateff 1924, Golemansky 1968, Golemansky et al. 2006); Rila Mt. (Todorov and Golemansky 2000, Todorov 2004, Todorov 2005, new data); Stara Planina Mt. (new data); Vitosha Mt. (Pateff 1924, Golemansky 1965, Golemansky and Todorov 1985, Golemansky and Todorov 1990, Todorov 1993, Todorov and Golemansky 1995, new data).

Notes: Besides the nominal species, two infrasubspecific taxa have also been recorded: Arcella discoides var. pseudovulgaris Deflandre, 1928 (Vitosha Mt.) and Arcella discoides var. scutelliformis Playfair, 1918 (Pirin Mt., Rhodopes Mt., Rila Mt.).

\section{Arcella gibbosa Penard, 1890}

Distribution: Rhodopes Mt. (Golemansky et al. 2006); Vitosha Mt. (Golemansky and Todorov 1985, Golemansky and Todorov 1990, Todorov 1993,Todorov and Golemansky 1995, new data). 
Arcella hemisphaerica Perty, 1852

Nomenclature:

Arcella vulgaris var. hemisphaerica Wailes, 1918

Distribution: Pirin Mt. (new data); Rhodopes Mt. (Golemansky 1968, Golemansky et al. 2006); Rila Mt. (Todorov 2004, new data); Vitosha Mt. (new data).

Notes: The species has been recorded both as nominal species and as infrasubspecific taxon Arcella hemisphaerica f. undulata Deflandre, 1928 (Rhodopes Mt., Rila Mt.).

Arcella intermedia (Deflandre, 1928) Tsyganov and Mazei, 2006

Nomenclature:

Arcella hemisphaerica var. intermedia f. undulata Deflandre, 1928

Distribution: Pirin Mt. (new data); Rila Mt. (new data); Stara Planina Mt. (new data); Vitosha Mt. (new data).

\section{Arcella rotundata Playfair, 1918}

Distribution: Pirin Mt. (Golemansky 1974, new data); Rhodopes Mt. (Golemansky et al. 2006); Rila Mt. (Todorov and Golemansky 2000, Todorov 2004, new data); Stara Planina Mt. (new data); Vitosha Mt. (Golemansky and Todorov 1985, Golemansky and Todorov 1990, Todorov 1993, Todorov and Golemansky 1995, new data).

Notes: Besides the nominal species, two infrasubspecific taxa have also been recorded: Arcella rotundata var. stenostoma Deflandre, 1928 (Vitosha Mt.) and Arcella rotundata var. stenostoma f. undulata Deflandre, 1928 (Rhodopes Mt., Vitosha Mt.).

\section{Arcella vulgaris Ehrenberg, 1830}

Distribution: Rhodopes Mt. (Pateff 1924, Golemansky et al. 2006); Rila Mt. (Todorov and Golemansky 2000); Vitosha Mt. (Golemansky and Todorov 1985,Golemansky and Todorov 1990, Todorov 1993, Todorov and Golemansky 1995, new data).

Notes: The species has been recorded both as nominal species and as infrasubspecific taxon Arcella vulgaris f. undulata Deflandre, 1928 (Rila Mt.).

Family Netzeliidae Kosakyan, Lara, Gomaa and Lahr, 2016 


\section{Genus Netzelia Ogden, 1979}

Netzelia tuberculata (Wallich, 1864) Netzel, 1983

Nomenclature:

Difflugia proteiformis subsp. globularis var. tuberculata Wallich, 1864

Difflugia tuberculata Archer, 1867

Difflugia lobostoma Leidy, 1879 (in part)

Nebela tuberculata Owen and Jones, 1976

Difflugia tricuspis Medioli and Scott, 1983 (in part)

Distribution: Pirin Mt. (new data); Rhodopes Mt. (Golemansky 1968, Golemansky et al. 2006); Rila Mt. (Todorov 2004).

Notes: The species has been recorded both as nominal species and as synonym $D$. tuberculata (Rhodopes Mt.).

Suborder Difflugina Meisterfeld, 2002

Family Difflugiidae Wallich, 1864

Genus Difflugia Leclerc, 1815

Difflugia acuminata Ehrenberg, 1838

Nomenclature:

Difflugia curvicaulis Penard, 1899

Difflugia acuminata var. umbilicata Penard, 1902

Difflugia venusta Ogden, 1983

Distribution: Pirin Mt. (new data); Rhodopes Mt. (Pateff 1924, Golemansky 1968, Golemansky et al. 2006); Rila Mt. (Todorov and Golemansky 2000, Todorov 2004, Todorov 2005, new data); Vitosha Mt. (Pateff 1924, new data).

Notes: The species has been recorded both as nominal species and as synonym $D$. venusta (Rhodopes Mt.).

\section{Difflugia ampullula Playfair, 1918}

Distribution: Pirin Mt. (new data); Rila Mt. (Todorov 2005, new data); Vitosha Mt. (new data). 


\section{Difflugia angulostoma Gauthier-Lièvre and Thomas, 1958}

Distribution: Rila Mt. (Todorov and Golemansky 2000); Stara Planina Mt. (new data).

\section{Difflugia bacillariarum Perty, 1849}

Nomenclature:

Difflugia bicornis Penard, 1890

Difflugia australis var. minor Gauthier-Lièvre et Thomas, 1958

Distribution: Stara Planina Mt. (new data); Rila Mt. (Golemansky and Todorov 1993).

\section{Difflugia brevicolla Cash, 1909}

Nomenclature:

Difflugia pyriformis var. atricolor Penard, 1902

Distribution: Rhodopes Mt. (Golemansky et al. 2006); Rila Mt. (new data); Stara Planina Mt. (new data).

Difflugia bryophila (Penard, 1902) Jung, 1942

Nomenclature:

Difflugia pyriformis var. bryophila Penard, 1902

Difflugia oblonga var. longicollis Gassowsky, 1936

Difflugia longicollis (Gassowsky, 1936) Ogden and Hedley, 1980

Difflugia gassowskii Ogden, 1983

Distribution: Pirin Mt. (new data); Rhodopes Mt. (Golemansky et al. 2006); Rila Mt. (Todorov and Golemansky 2000, Todorov 2004, Todorov 2005, new data); Stara Planina Mt. (new data); Vitosha Mt. (Golemansky and Todorov 1985, Golemansky and Todorov 1990, Todorov 1993, Todorov and Golemansky 1995, new data).

Notes: The species has been recorded both as nominal species and as synonym $D$. gassowskii (Rhodopes Mt., Rila Mt., Vitosha Mt.).

\section{Difflugia distenda Ogden, 1983}

Nomenclature:

Difflugia acuminata var. inflata Penard, 1899

Difflugia bicruris Gauthier-Lièvre et Thomas, 1958.

Distribution: Pirin Mt. (new data); Vitosha Mt. (Todorov 1993, Todorov and Golemansky 1995). 


\section{Difflugia elegans Penard, 1890}

Nomenclature:

Difflugia amphoralis Hopkinson, 1909

Difflugia australis (Playfair, 1918) Gauthier-Lièvre et Thomas, 1958

Difflugia borodini Gassowsky, 1936

Difflugia elegans f. bicornis Jung, 1936

Difflugia elegans f. tricornis Jung, 1936

Difflugia juzephiniensis Dekhtyar, 1993

Difflugia leidyi Wailes, 1912

Difflugia Solowetskii Mereschkowsky, 1877

Difflugia tricornis (Jung, 1936) Ogden, 1983

Difflugia varians Penard, 1902

Distribution: Pirin Mt. (Golemansky 1974, new data); Rhodopes Mt. (Golemansky 1968, Golemansky et al. 2006); Rila Mt. (Pateff 1924, Todorov and Golemansky 2000, Todorov 2004, Todorov 2005, new data); Stara Planina Mt. (new data); Vitosha Mt. (Pateff 1924, Golemansky 1965, Todorov 1993, Todorov and Golemansky 1995, new data).

Notes: The species has been recorded both as nominal species and as synonym $D$. amphoralis (Rila Mt., Vitosha Mt.).

\section{Difflugia glans Penard, 1902}

Distribution: Rila Mt. (Todorov and Golemansky 2000).

\section{Difflugia globulosa Dujardin, 1837}

Nomenclature:

Difflugia proteiformis Ehrenberg, 1838 subsp. globularis Wallich, 1864

Difflugia globularis (Wallich, 1864) Leidy, 1877

Difflugia chardezi Godeanu, 1972

Distribution: Pirin Mt. (new data); Rhodopes Mt. (Golemansky et al. 2006); Rila Mt. (Todorov and Golemansky 2000, Todorov 2004, Todorov 2005, new data); Stara Planina Mt. (new data); Vitosha Mt. (Todorov 1993, Golemansky and Todorov 1985, Golemansky and Todorov 1990, Todorov and Golemansky 1995, new data).

Notes: The species has been recorded both as nominal species and as synonym $D$. globularis (Rhodopes Mt., Rila Mt., Vitosha Mt.). 


\section{Difflugia hiraethogii Ogden, 1983}

Distribution: Pirin Mt. (new data); Rila Mt. (Todorov and Golemansky 2000, Todorov 2004, Todorov 2005, new data); Stara Planina Mt. (new data); Vitosha Mt. (new data).

\section{Difflugia lanceolata Penard, 1890}

Distribution: Pirin Mt. (new data); Rhodopes Mt. (Golemansky 1968, Golemansky et al. 2006); Rila Mt. (Todorov and Golemansky 2000, new data); Stara Planina Mt. (new data); Vitosha Mt. (Golemansky 1965, Golemansky and Todorov 1990).

\section{Difflugia lobostoma Leidy, 1874}

Distribution: Rhodopes Mt. (Golemansky 1968, Golemansky et al. 2006)

\section{Difflugia lucida Penard, 1890}

Distribution: Pirin Mt. (Golemansky 1974, new data); Rhodopes Mt. (Golemansky et al. 2006); Rila Mt. (Golemansky and Todorov 1993, Todorov and Golemansky 2000, Todorov 2004, Todorov 2005, new data); Stara Planina Mt. (new data); Vitosha Mt. (Golemansky 1965, Golemansky and Todorov 1985, Golemansky and Todorov 1990, Todorov 1993, Todorov and Golemansky 1995, new data).

\section{Difflugia mammillaris Penard, 1893}

Distribution: Rila Mt. (Todorov 2004).

Difflugia mica Frenzel, 1892

Distribution: Rila Mt. (Todorov 2005); Vitosha Mt. (new data).

Difflugia microclaviformis (Kourov, 1925) Ogden, 1983

Nomenclature:

Difflugia pyriformis var. venusta Penard 1902

Difflugia oblogna var. venusta Cash, 1909

Difflugia oblonga var. microclaviformis Kourov, 1925

Distribution: Vitosha Mt. (Golemansky 1965, Golemansky and Todorov 1990).

Notes: The species has been recorded both as nominal species and as the infrasubspecific taxon Difflugia oblonga var. microclaviformis (Vitosha Mt.). 


\section{Difflugia minuta Rampi, 1950}

Distribution: Pirin Mt. (new data); Rila Mt. (Todorov 2004, Todorov 2005); Vitosha Mt. (new data).

\section{Difflugia molesta Penard, 1902}

Nomenclature:

Difflugia levanderi Playfair, 1918

Distribution: Rhodopes Mt. (Golemansky et al. 2006); Rila Mt. (Todorov 2004, Todorov 2005, new data); Vitosha Mt. (new data).

\section{Difflugia oblonga Ehrenberg, 1838}

Nomenclature:

Difflugia bacillifera Penard, 1890

Difflugia lacustris (Penard, 1899) Ogden, 1983

Difflugia oblonga f. cyphodera Jung, 1942

Difflugia oblonga var. incondita Gauthier-Lièvre et Thomas, 1958

Difflugia oblonga var. lacustris Cash and Hopkinson, 1909

Difflugia oblonga var. parva Thomas, 1954

Difflugia parva (Thomas, 1954) Ogden, 1983

Difflugia pyriformis var. lacustris Penard, 1899

Distribution: Pirin Mt. (new data); Rhodopes Mt. (Golemansky 1968, Golemansky et al. 2006); Rila Mt. (Pateff 1924, Todorov and Golemansky 2000, Todorov 2005, new data); Stara Planina Mt. (new data); Vitosha Mt. (Pateff 1924, Golemansky 1965, Golemansky and Todorov 1985, Golemansky and Todorov 1990, Todorov 1993, Todorov and Golemansky 1995, new data).

Notes: Besides the nominal species, the synonyms D. parva (Rhodopes Mt., Rila Mt., Vitosha Mt.), D. lacustris (Rhodopes Mt., Vitosha Mt.), D. bacillifera (Rila Mt., Vitosha Mt.) and infrasubspecific taxa $D$. oblonga var. lacustris (Vitosha Mt.) and $D$. oblonga var. parva (Rila Mt., Vitosha Mt.) have also been recorded.

\section{Difflugia penardi Hopkinson, 1909}

Nomenclature:

Difflugia fallax Penard, 1890

Difflugia pyriformis var. tenuis Penard, 1890

Difflugia manicata Penard, 1902 
Difflugia oblonga var. tenuis Wailes and Penard, 1911

Difflugia tenuis (Penard, 1890) Ogden, 1983

Distribution: Pirin Mt. (Golemansky 1974, new data); Rhodopes Mt. (Golemansky 1968, Golemansky et al. 2006); Rila Mt. (Todorov and Golemansky 2000, Todorov 2004, new data); Stara Planina Mt. (new data); Vitosha Mt. (Golemansky 1965, Golemansky and Todorov 1990, Todorov 1993, Todorov and Golemansky 1995, new data).

Notes: The species has been recorded both as nominal species and as synonym $D$. manicata (Rhodopes Mt., Rila Mt., Vitosha Mt.).

\section{Difflugia petricola Cash, 1909}

Distribution: Pirin Mt. (new data); Rhodopes Mt. (Golemansky et al. 2006); Rila Mt. (Todorov 2004, Todorov 2005).

\section{Difflugia pristis Penard, 1902}

Distribution: Pirin Mt. (new data); Rhodopes Mt. (Golemansky et al. 2006); Rila Mt. (Todorov and Golemansky 2000, Todorov 2004, Todorov 2005, new data); Stara Planina Mt. (new data); Vitosha Mt. (new data).

\section{Difflugia pulex Penard, 1902}

Nomenclature:

Difflugia minuta var. minor Godeanu, 1972

Difflugia ovalisina Beyens et Chardez, 1994

Distribution: Pirin Mt. (new data); Rhodopes Mt. (Golemansky et al. 2006); Rila Mt. (Pateff 1924, Todorov and Golemansky 2000, Todorov 2004, Todorov 2005, new data); Stara Planina Mt. (new data); Vitosha Mt. (Golemansky and Todorov 1990, Todorov 1993, Todorov and Golemansky 1995, new data).

\section{Difflugia pyriformis Perty, 1849}

Distribution: Rhodopes Mt. (Pateff 1924); Rila Mt. (Pateff 1924); Vitosha Mt. (Pateff 1924).

\section{Difflugia rotunda (Chardez, 1956) Ogden, 1983}

\section{Nomenclature:}

Difflugia globularis var. sphaerica Chardez, 1956

Distribution: Rila Mt. (Todorov 2004). 


\section{Difflugia rubescens Penard, 1891}

Distribution: Pirin Mt. (Golemansky 1974, new data); Rila Mt. (Pateff 1924, Golemansky and Todorov 1993, new data); Stara Planina Mt. (new data); Vitosha Mt. (Golemansky 1965, Golemansky and Todorov 1985, Golemansky and Todorov 1990, Todorov 1993, Todorov and Golemansky 1995, new data).

Difflugia stoutii Ogden, 1983

Distribution: Rila Mt. (Todorov and Golemansky 2000).

Difflugia subaequalis Penard, 1910

Distribution: Pirin Mt. (Golemansky 1974).

\section{Difflugia urceolata Carter, 1864}

Nomenclature:

Difflugia urceolata var. olla Leidy, 1879

Difflugia urceolata var. sphaerica Playfair, 1917

Distribution: Pirin Mt. (new data); Rhodopes Mt. (Pateff 1924, Golemansky et al. 2006).

Difflugia ventricosa Deflandre, 1926

Distribution: Rhodopes Mt. (Golemansky et al. 2006).

Difflugia viscidula Penard, 1902

Nomenclature:

Difflugia lemani Blanc, 1892

Difflugia histrio Penard, 1908

Difflugia lemani var. palustris Chardez, 1956

Difflugia lebes var. masurica Schönborn, 1965

Difflugia lebes var. bretschkoi Laminger, 1971

Difflugia finstertaliensis Laminger, 1971

Distribution: Pirin Mt. (new data); Rhodopes Mt. (Golemansky et al. 2006); Rila Mt. (Todorov and Golemansky 2000, Todorov 2004, Todorov 2005, new data); Vitosha Mt. (Pateff 1928, Golemansky 1965, Golemansky and Todorov 1985, Golemansky and Todorov 1990). 
Notes: The species has been recorded as nominal species, as synonym $D$. lemani ( Vitosha Mt.), as infrasubspecific taxon $D$. lemani var. palustris (Vitosha Mt.) and as Difflugia sp. (Vitosha Mt.).

Genus Lagenodifflugia Medioli and Scott, 1983

Lagenodifflugia bryophila (Penard, 1902) Ogden, 1987

Nomenclature:

Pontigulasia bryophila Penard, 1902

Pontigulasia bryophila var. elachys Jung, 1942

Pontigulasia varadi Godeanu, 1972

Zivkovicia bryophila (Penard, 1902) Ogden, 1983

Distribution: Pirin Mt. (Golemansky 1974, new data); Rhodopes Mt. (Pateff 1924, Golemansky et al. 2006); Rila Mt. (Pateff 1924, Todorov and Golemansky 2000, Todorov 2004, Todorov 2005, new data); Stara Planina Mt. (new data); Vitosha Mt. (Pateff 1924, Golemansky 1965, Golemansky and Todorov 1990, Todorov 1993, Todorov and Golemansky 1995, new data).

Notes: The species has been recorded both as nominal species and as synonym $P$. bryophila (Rhodopes Mt., Rila Mt., Vitosha Mt.).

Lagenodifflugia montana (Ogden and Zivkovic, 1983) Ogden, 1987

Nomenclature:

Pontigulasia montana Ogden and Zivkovic, 1983

Distribution: Pirin Mt. (new data); Rila Mt. (new data).

Lagenodifflugia vas (Leidy, 1874) Medioli and Scott, 1983

Nomenclature:

Difflugia vas Leidy, 1874

Difflugia pyriformis var. vas Leidy, 1879

Pontigulasia vas (Leidy) Schouteden, 1906

Zivkovicia vas (Leidy, 1874) Ogden, 1983 (in part)

Distribution: Pirin Mt. (new data); Rhodopes Mt. (Golemansky et al. 2006); Rila Mt. (new data); Vitosha Mt. (new data). 
Genus Pontigulasia Rhumbler, 1896

Pontigulasia elisa (Penard, 1893) Schouteden, 1906

Nomenclature:

Difflugia elisa Penard, 1893

Pontigulasia incisa Rhumbler, 1896

Distribution: Pirin Mt. (new data); Rila Mt. (Golemansky and Todorov 1993); Vitosha Mt. (Todorov 1993, Todorov and Golemansky 1995).

Pontigulasia rhumbleri Hopkinson, 1920

Nomenclature:

Pontigulasia compressa Rhumbler, 1896

(non Pontigulasia compressa (Carter, 1864))

Distribution: Pirin Mt. (new data); Rhodopes Mt. (Pateff 1924, Golemansky et al. 2006); Rila Mt. (Pateff 1924, Todorov 2004, Todorov 2005); Stara Planina Mt. (new data); Vitosha Mt. (Pateff 1924).

Notes: The species has been recorded both as nominal species and as synonym $P$. compressa (Rhodopes Mt., Rila Mt., Vitosha Mt.).

\section{Genus Zivkovicia Ogden, 1987}

\section{Zivkovicia compressa (Carter, 1864)}

Nomenclature:

Difflugia compressa Carter, 1864

Difflugia pyriformis var. vas sub- var. bigibbosa Penard, 1899

Pontigulasia bigibbosa Penard, 1902

Distribution: Pirin Mt. (Golemansky 1974, new data); Rhodopes Mt. (Golemansky et al. 2006); Rila Mt. (Todorov and Golemansky 2000, Todorov 2004, Todorov 2005, new data); Stara Planina Mt. (new data); Vitosha Mt. (Golemansky 1965, Golemansky and Todorov 1985, Golemansky and Todorov 1990, Todorov 1993, Todorov and Golemansky 1995, new data).

Notes: The species has been recorded both as nominal species and as synonym $P$. bigibbosa (Pirin Mt., Vitosha Mt.). Furthermore, in several publications on the testate amoebae from Vitosha Mt. (Golemansky 1965, Golemansky and Todorov 1990, Todorov 1993, Todorov and Golemansky 1995), the infrasubspecific taxa Difflugia compressa var. africana Gauthier-Lièvre et Thomas, 1958 has erroneously been 
recorded, because the description of the found individuals fully corresponds to $Z$. compressa.

Zivkovicia spectabilis (Penard, 1902) Ogden, 1987

Nomenclature:

Pontigulasia spectabilis Penard, 1902

Zivkovicia vas (Leidy, 1874) Ogden, 1983 (in part)

Distribution: Rhodopes Mt. (Golemansky 1968); Rila Mt. (Todorov 2004).

Notes: The species has been recorded both as nominal species and as synonym $P$. spectabilis (Rhodopes Mt.).

Family Centropyxidae Jung, 1942

Genus Centropyxis Stein, 1857

Centropyxis aculeata (Ehrenberg, 1830) Stein, 1857

Nomenclature:

Arcella aculeata Ehrenberg, 1830

Difflugia aculeata Perty, 1852

Echinopyxis aculeata Claparède et Lachmann, 1859

Distribution: Pirin Mt. (Golemansky 1974, new data); Rhodopes Mt. (Pateff 1924, Golemansky 1968, Golemansky et al. 2006); Rila Mt. (Golemansky and Todorov 1993, Todorov and Golemansky 2000, Todorov 2004, Todorov 2005, new data); Stara Planina Mt. (new data); Vitosha Mt. (Pateff 1924, Golemansky 1965, Golemansky and Todorov 1985, Golemansky and Todorov 1990, Todorov 1993, Todorov and Golemansky 1995, new data).

Notes: Besides the nominal species, two infrasubspecific taxa Centropyxis aculeata var. oblonga Deflandre, 1929 (Rhodopes Mt., Rila Mt., Vitosha Mt.) and Centropyxis aculeata var. grandis Deflandre, 1929 (Vitosha Mt.) have also been recorded.

\section{Centropyxis aerophila Deflandre, 1929}

Nomenclature:

Difflugia constricta Ehrenberg, 1838

Arcella arctiscon Ehrenberg, 1854

Distribution: Pirin Mt. (Golemansky 1974, new data); Rhodopes Mt. (Golemansky 1968, Golemansky et al. 2006); Rila Mt. (Golemansky and Todorov 1993, Todorov and 
Golemansky 2000, Todorov 2004, Todorov 2005, new data); Stara Planina Mt. (new data); Vitosha Mt. (Golemansky 1965, Golemansky and Todorov 1985, Golemansky and Todorov 1990, Todorov 1993, Todorov and Golemansky 1995, new data).

Notes: Besides the nominal species, the infrasubspecific taxon Centropyxis aerophila var. sphagnicola Deflandre, 1929 has also been recorded (Rila Mt., Vitosha Mt.).

Centropyxis cassis (Wallich, 1864) Deflandre, 1929

Nomenclature:

Difflugia cassis Wallich, 1864

Distribution: Pirin Mt. (new data); Rhodopes Mt. (Golemansky et al. 2006); Rila Mt. (Todorov 2004, Todorov 2005, Todorov and Golemansky 2000, new data); Stara Planina Mt. (new data); Vitosha Mt. (Golemansky 1965, Golemansky and Todorov 1985, Golemansky and Todorov 1990, Todorov 1993, Todorov and Golemansky 1995, new data).

Centropyxis constricta (Ehrenberg, 1838) Penard, 1902

Nomenclature:

Difflugia constricta Ehrenberg, 1838

Arcella constricta Ehrenberg, 1841

Distribution: Pirin Mt. (Golemansky 1974, new data); Rhodopes Mt. (Pateff 1924, Golemansky et al. 2006); Rila Mt. (Pateff 1924, new data); Stara Planina Mt. (new data); Vitosha Mt. (Pateff 1924, Golemansky 1965, Golemansky and Todorov 1990, new data).

Notes: The species has been recorded both as nominal species and as synonym $D$. constricta (Rhodopes Mt., Rila Mt., Vitosha Mt.).

Centropyxis cryptostoma Bonnet, 1959

Distribution: Rila Mt. (new data).

Centropyxis discoides (Penard, 1890) Deflandre, 1929

Nomenclature:

Centropyxis aculeata var. discoides Penard, 1890

Distribution: Pirin Mt. (new data); Rhodopes Mt. (Golemansky et al. 2006); Rila Mt. (Todorov 2005, new data); Stara Planina Mt. (new data); Vitosha Mt. (Golemansky 1965, Golemansky and Todorov 1990, new data). 
Centropyxis ecornis (Ehrenberg, 1841) Leidy, 1879

Nomenclature:

Arcella ecornis Ehrenberg, 1841

Distribution: Pirin Mt. (Golemansky 1974, new data); Rhodopes Mt. (Golemansky et al. 2006); Rila Mt. (Todorov and Golemansky 2000, Todorov 2004, Todorov 2005, new data); Stara Planina Mt. (new data); Vitosha Mt. (Todorov 1993, Todorov and Golemansky 1995, new data).

Centropyxis elongata (Penard, 1890) Thomas, 1959

Nomenclature:

Difflugia constricta var. elongata Penard, 1890

Distribution: Pirin Mt. (Golemansky 1974, new data); Rhodopes Mt. (Golemansky et al. 2006); Rila Mt. (Todorov and Golemansky 2000, new data); Vitosha Mt. (Golemansky and Todorov 1985, Golemansky and Todorov 1990, Todorov 1993, Todorov and Golemansky 1995).

Centropyxis gibba Deflandre, 1929

Distribution: Rhodopes Mt. (Golemansky et al. 2006); Rila Mt. (Todorov 2005).

Centropyxis hirsuta Deflandre, 1929

Distribution: Rila Mt. (Todorov 2004).

Centropyxis laevigata Penard, 1890

Distribution: Rhodopes Mt. (Golemansky et al. 2006).

Centropyxis minuta Deflandre, 1929

Nomenclature:

Difflugia constricta p. p. Leidy, 1879, PL. XVIII, figs. 15-16

Difflugia constricta p. p. Penard, 1902, p. 299, figs. 13-14

Distribution: Rhodopes Mt. (Golemansky et al. 2006).

Centropyxis orbicularis Deflandre, 1929

Distribution: Pirin Mt. (new data); Rila Mt. (new data); Stara Planina Mt. (new data). 
Centropyxis plagiostoma Bonnet and Thomas, 1955

Distribution: Rhodopes Mt. (Golemansky et al. 2006); Rila Mt. (Golemansky and Todorov 1993).

Centropyxis platystoma (Penard, 1890) Deflandre, 1929

Nomenclature:

Difflugia platystoma Penard, 1890

Difflugia constricta p. p. Leidy, 1879, PL. XVIII, figs. 20-21

Difflugia constricta p. p. Penard, 1902, p. 299, figs. 8, 11, 12

Distribution: Pirin Mt. (new data); Rhodopes Mt. (Golemansky et al. 2006); Rila Mt. (Todorov and Golemansky 2000, Todorov 2005, new data); Stara Planina Mt. (new data); Vitosha Mt. (Golemansky 1965, Golemansky and Todorov 1985, Golemansky and Todorov 1990, Todorov 1993, Todorov and Golemansky 1995, new data).

Notes: Besides the nominal species, the infrasubspecific taxon Centropyxis platystoma var. armata Deflandre, 1929 has also been recorded (Vitosha Mt.).

Centropyxis spinosa (Cash, 1905) Deflandre, 1929

Nomenclature:

Centropyxis aculeata var. spinosa Cash, 1905

Distribution: Pirin Mt. (new data); Vitosha Mt. (Todorov 1993, Todorov and Golemansky 1995).

Centropyxis sylvatica (Deflandre, 1929) Bonnet and Thomas, 1955

Nomenclature:

Centropyxis aerophila var. sylvatica Deflandre, 1929

Distribution: Pirin Mt. (Golemansky 1974, new data); Rhodopes Mt. (Golemansky et al. 2006); Rila Mt. (Golemansky and Todorov 1993, Todorov 2004, Todorov 2005, new data); Stara Planina Mt. (new data); Vitosha Mt. (Golemansky 1965, Golemansky and Todorov 1985, Golemansky and Todorov 1990, Todorov 1993, Todorov and Golemansky 1995, new data). 
Family Trigonopyxidae Loeblich and Tappan, 1964

Genus Cyclopyxis Deflandre, 1929

Cyclopyxis arcelloides (Penard, 1902) Deflandre, 1929

Nomenclature:

Centropyxis arceloides Penard, 1902

Distribution: Vitosha Mt. (Pateff 1928, Golemansky 1965, Golemansky and Todorov 1990, new data).

Notes: The species is recorded both as nominal species and as synonym Centropyxis arceloides (Vitosha Mt.).

Cyclopyxis eurystoma Deflandre, 1929

Nomenclature:

Centropyxis (Cyclopyxis) eurystoma Deflandre, 1929

Distribution: Pirin Mt. (Golemansky 1974, new data); Rhodopes Mt. (Golemansky 1968, Golemansky et al. 2006); Rila Mt. (Golemansky and Todorov 1993, Todorov and Golemansky 2000, new data); Stara Planina Mt. (new data); Vitosha Mt. (Golemansky 1965, Golemansky and Todorov 1985, Golemansky and Todorov 1990, Todorov 1993, Todorov and Golemansky 1995, new data).

\section{Cyclopyxis kahli Deflandre, 1929}

Nomenclature:

Centropyxis (Cyclopyxis) kahli Deflandre, 1929

Distribution: Pirin Mt. (Golemansky 1974, new data); Rhodopes Mt. (Golemansky et al. 2006); Rila Mt. (Golemansky and Todorov 1993, Todorov and Golemansky 2000, new data); Stara Planina Mt. (new data); Vitosha Mt. (Golemansky 1965, Golemansky and Todorov 1985, Golemansky and Todorov 1990, new data).

\section{Cyclopyxis pirini Golemansky, 1974}

Distribution: Pirin Mt. (Golemansky 1974); Vitosha Mt. (Golemansky and Todorov 1985, Golemansky and Todorov 1990, Todorov 1993, Todorov and Golemansky 1995, new data).

Cyclopyxis puteus Thomas, 1960

Distribution: Rila Mt. (new data). 
Genus Trigonopyxis Penard, 1912

Trigonopyxis arcula (Leidy, 1879) Penard, 1912

Nomenclature:

Difflugia arcula Leidy, 1879

Cystidina arcula (Leidy, 1879) Volz, 1929

Distribution: Rila Mt. (new data); Stara Planina Mt. (new data); Vitosha Mt. (Golemansky 1965, Golemansky and Todorov 1990, new data).

Family Plagiopyxidae Bonnet and Thomas, 1960

Genus Bullinularia Deflandre, 1953

Bullinularia indica (Penard, 1907) Deflandre, 1953

Nomenclature:

Bulinella indica Penard, 1907

Bullinula indica Penard, 1912

Distribution: Pirin Mt. (new data); Rila Mt. (new data).

Genus Plagiopyxis Penard, 1910

Plagiopyxis callida Penard, 1910

Nomenclature:

Bullinula indica var. callida Jung, 1936

Distribution: Pirin Mt. (Golemansky 1974, new data); Rila Mt. (new data); Stara Planina Mt. (new data); Vitosha Mt. (new data).

Plagiopyxis declivis Thomas, 1955

Distribution: Pirin Mt. (new data); Rhodopes Mt. (Golemansky et al. 2006); Rila Mt. (Todorov and Golemansky 2000, new data); Stara Planina Mt. (new data); Vitosha Mt. (Todorov 1993, Todorov and Golemansky 1995, new data).

Plagiopyxis glyphostoma Bonnet, 1959

Distribution: Vitosha Mt. (Todorov 1993, Todorov and Golemansky 1995). 
Plagiopyxis labiata Penard, 1910

Nomenclature:

Centropyxia labiata Bartoš, 1947

Distribution: Pirin Mt. (Golemansky 1974); Rila Mt. (Golemansky and Todorov 1993).

Plagiopyxis minuta Bonnet, 1959

Distribution: Vitosha Mt. (Todorov 1993, Todorov and Golemansky 1995).

Plagiopyxis oblonga (Bonnet et Thomas, 1955)

Distribution: Stara Planina Mt. (new data).

Family Hyalospheniidae (Schulze, 1877) Kosakyan et Lara, 2012

Genus Hyalosphenia (Stein, 1857) Schulze, 1877

Hyalosphenia elegans (Leidy, 1874) Leidy, 1879

Nomenclature:

Difflugia (Catharia) elegans Leidy, 1874

Hyalosphenia turfacea Taránek, 1881

Distribution: Vitosha Mt. (Todorov 1993, Todorov and Golemansky 1995).

Hyalosphenia papilio (Leidy, 1874) Leidy, 1875

Nomenclature:

Difflugia (Catharia) papilio Leidy, 1874

Distribution: Pirin Mt. (new data); Rhodopes Mt. (Golemansky 1968, Golemansky et al. 2006); Rila Mt. (Golemansky and Todorov 1993, Todorov and Golemansky 2000,Todorov 2005, new data); Stara Planina Mt. (new data); Vitosha Mt. (Pateff 1924, Golemansky 1965,Golemansky and Todorov 1985, Golemansky and Todorov 1990, Todorov 1993, Todorov and Golemansky 1995, new data).

Genus Nebela (Leidy, 1874) sensu Kosakyan, Lahr, Mulot, Meisterfeld, Mitchell and Lara, 2016

Nebela aliciae Mitchell et Lara, 2013

Distribution: Stara Planina Mt. (new data); Vitosha Mt. (new data). 
Nebela collaris (Ehrenberg 1848) Leidy, 1879

Nomenclature:

Difflugia collaris Ehrenberg 1848

Diffluga cancellata Ehrenberg 1848

Difflugia reticulata Ehrenberg 1848

Difflugia carpio Ehrenberg 1854

Difflugia laxa Ehrenberg 1871

Difflugia cellulifera Ehrenberg 1874

Nebela numata Leidy 1874

Nebela bohemica Taranek 1882

Nebela sphagnophila (Steinecke) Van Oye 1933

Nebela tincta var. major Deflandre 1936

Nebela tincta f. stenostoma Jung 1936

Distribution: Pirin Mt. (Golemansky 1974, new data); Rhodopes Mt. (Pateff 1924, Golemansky 1968, Golemansky et al. 2006); Rila Mt. (Golemansky and Todorov 1993, Todorov and Golemansky 2000, Todorov 2005, new data); Stara Planina Mt. (new data); Vitosha Mt. (Pateff 1924, Golemansky 1965, Golemansky and Todorov 1985, Golemansky and Todorov 1990, Todorov 1993, Todorov and Golemansky 1995, Kosakyan et al. 2012; new data).

Notes: The species has been recorded as nominal species, as synonym $N$. bohemica ( Pirin Mt., Rhodopes Mt., Rila Mt., Vitosha Mt.) and as infrasubspecific taxon Nebela tincta var. major (Vitosha Mt.).

\section{Nebela flabellulum Leidy, 1874}

Nomenclature:

Difflugia (Nebela) flabellulum Leidy, 1874

Distribution: Rhodopes Mt. (Golemansky 1968, Golemansky et al. 2006); Stara Planina Mt. (new data).

\section{Nebela guttata Kosakyan et Lara, 2013}

Distribution: Pirin Mt. (new data); Rila Mt. (new data); Stara Planina Mt. (new data); Vitosha Mt. (new data). 
Nebela militaris Penard, 1890

Distribution: Pirin Mt. (new data); Rila Mt. (Pateff 1924, new data); Stara Planina Mt. (new data); Vitosha Mt. (Pateff 1924, Golemansky 1965, Golemansky and Todorov 1990, Todorov 1993, Todorov and Golemansky 1995, new data).

Nebela pechorensis Kosakyan et Mitchell, 2013

Distribution: Pirin Mt. (new data); Rila Mt. (new data); Stara Planina Mt. (new data); Vitosha Mt. (new data).

Nebela rotunda Penard, 1890

Nomenclature:

Nebela tincta var. rotunda Penard, 1890

Distribution: Vitosha Mt. (new data).

Nebela tincta (Leidy, 1879) Awerintzew, 1906

Nomenclature:

Hyalosphenia tincta Leidy, 1879

Nebela bursella Vejdovsky, 1882

Nebela minor Penard, 1902

Nebela parvula Cash, 1909

Distribution: Pirin Mt. (Golemansky 1974, new data); Rhodopes Mt. (Golemansky 1968, Golemansky et al. 2006); Rila Mt. (Pateff 1924, Golemansky and Todorov 1993, Todorov and Golemansky 2000, new data); Stara Planina Mt. (new data); Vitosha Mt. (Pateff 1924, Golemansky 1965, Golemansky and Todorov 1985, Golemansky and Todorov 1990, Todorov 1993, Todorov and Golemansky 1995, new data).

Notes: The species has been recorded both as nominal species and as synonyms $N$. bursella (Rila Mt, Vitosha Mt.), N. minor (Rhodopes Mt., Rila Mt.) and N. parvula ( Pirin Mt., Rhodopes Mt., Vitosha Mt.).

Genus Gibbocarina Kosakyan, Lahr, Mulot, Meisterfeld, Mitchell and Lara, 2016

Gibbocarina galeata (Penard, 1890) Kosakyan, Lahr, Mulot, Meisterfeld, Mitchell and Lara, 2016

Nomenclature:

Nebela galeata Penard, 1890 
Distribution: Pirin Mt. (new data); Rhodopes Mt. (Pateff 1924, Golemansky 1968, Golemansky et al. 2006); Rila Mt. (Pateff 1924, Todorov and Golemansky 2000, Todorov 2005, new data); Stara Planina Mt. (new data); Vitosha Mt. (Pateff 1924, Golemansky 1965, Golemansky and Todorov 1985, Golemansky and Todorov 1990, Todorov 1993, Todorov and Golemansky 1995, new data).

Notes: So far, all records for the species have been as $N$. galeata.

Genus Longinebela Kosakyan, Lahr, Mulot, Meisterfeld, Mitchell and Lara, 2016 Longinebela ampulla Todorov, Bankov and Ganeva, 2018

Distribution: Stara Planina Mt. (Todorov et al. 2018).

Longinebela golemanskyi (Todorov, 2010) Kosakyan, Lahr, Mulot, Meisterfeld, Mitchell and Lara, 2016

Nomenclature:

Nebela golemanskyi Todorov, 2010

Distribution: Vitosha Mt. (Todorov 2010, new data).

Notes: The species has been recorded as N. golemanskyi.

Longinebela penardiana (Deflandre, 1936) Kosakyan, Lahr, Mulot, Meisterfeld, Mitchell and Lara, 2016

Nomenclature:

Nebela penardiana Deflandre, 1936

Distribution: Pirin Mt. (Golemansky 1974, new data); Rhodopes Mt. (Golemansky 1968, Golemansky et al. 2006); Rila Mt. (Golemansky and Todorov 1993, Todorov and Golemansky 2000, Todorov 2005, new data); Stara Planina Mt. (new data); Vitosha Mt. (Golemansky 1965, Golemansky and Todorov 1985, Golemansky and Todorov 1990, Todorov 1993, Todorov and Golemansky 1995, Kosakyan et al. 2012, new data).

Notes: So far, all records for the species have been as $N$. penardiana.

Longinebela speciosa (Deflandre, 1936) Kosakyan, Lahr, Mulot, Meisterfeld, Mitchell and Lara, 2016

Nomenclature:

Nebela speciosa Deflandre, 1936 
Distribution: Pirin Mt. (new data); Rhodopes Mt. (Golemansky et al. 2006); Rila Mt. (Todorov and Golemansky 2000, new data); Stara Planina Mt. (new data); Vitosha Mt. (Golemansky 1965, Golemansky and Todorov 1985, Golemansky and Todorov 1990, Todorov 1993, Todorov and Golemansky 1995, Kosakyan et al. 2012, new data).

Notes: So far, all records for the species have been as $N$. speciosa.

Longinebela tubulosa (Penard, 1902) Kosakyan, Lahr, Mulot, Meisterfeld, Mitchell and Lara, 2016

Nomenclature:

Nebela tubulosa Penard, 1902

Distribution: Pirin Mt. (new data); Rila Mt. (Todorov and Golemansky 2000, Todorov 2005, new data); Stara Planina Mt. (new data); Vitosha Mt. (Todorov 1993, Todorov and Golemansky 1995, Kosakyan et al. 2012, new data).

Notes: So far, all records for the species have been as $N$. tubulosa.

\section{Genus Padaungiella Lara et Todorov, 2012}

\section{Padaungiella americana (Taranek, 1882)}

Nomenclature:

Nebela americana Taranek, 1882.

Distribution: Rhodopes Mt. (Pateff 1924, Golemansky et al. 2006); Vitosha Mt. (Pateff 1924, Golemansky 1965, Golemansky and Todorov 1990).

Notes: All records for the species have been as N. americana (Rhodopes Mt., Vitosha Mt.). This species is with doubtful identity since it is sharing overlapping characters with $P$. lageniformis and with $P$. wailesi.

\section{Padaungiella lageniformis (Penard, 1890) Lara et Todorov, 2012}

Nomenclature:

Nebela lageniformis Penard, 1890

Distribution: Pirin Mt. (Golemansky 1974, new data); Rhodopes Mt. (Pateff 1924, Golemansky 1968, Golemansky et al. 2006); Rila Mt. (Pateff 1924, Golemansky and Todorov 1993, Todorov and Golemansky 2000, Todorov 2004, Todorov 2005, new data); Stara Planina Mt. (new data); Vitosha Mt. (Pateff 1924, Golemansky 1965, Golemansky and Todorov 1985, Golemansky and Todorov 1990, Todorov 1993, Todorov and Golemansky 1995, Kosakyan et al. 2012, new data). 
Notes: All records for the species (except these in Kosakyan et al. 2012) have been as N. lageniformis.

Padaungiella nebeloides (Gauthier-Lièvre and Thomas, 1958) Lara et Todorov, 2012

Nomenclature:

Difflugia nebeloides Gauthier-Lièvre and Thomas, 1958

Nebela nebeloides (Gauthier-Lièvre and Thomas, 1958) Todorov, Golemansky and Meisterfeld, 2010

Distribution: Pirin Mt. (new data); Rila Mt. (new data); Stara Planina Mt. (new data); Vitosha Mt. (Todorov et al. 2010, new data).

Notes: The species has been recorded by the synonymous name $N$. nebeloides. It should be noted that Mazei and Warren (2014) erroneously synonymised $D$. nebeloides with $D$. linearis (Penard, 1890) Gautier-Lièvre et Thomas, 1958, because they have not taken into account the fact that this species has recently been transferred from the genus Difflugia into the genus Nebela and subsequently in the newly described genus Padaungiella (Todorov et al. 2010, Kosakyan et al. 2012).

\section{Padaungiella tubulata (Brown, 1911) Lara et Todorov, 2012}

Nomenclature:

Nebela tubulata Brown, 1911

Distribution: Pirin Mt. (new data); Rhodopes Mt. (Golemansky et al. 2006); Rila Mt. (Todorov 2005, new data); Stara Planina Mt. (new data); Vitosha Mt. (Golemansky 1965, Golemansky and Todorov 1990, Todorov 1993, Todorov and Golemansky 1995, new data).

Notes: So far, all records for the species have been as $N$. tubulata.

\section{Padaungiella wailesi (Deflandre, 1936) Lara et Todorov, 2012}

Nomenclature:

Nebela wailesi Deflandre, 1936

Distribution: Rhodopes Mt. (Golemansky et al. 2006).

Notes: The species has been recorded by the synonymous name $N$. wailesi. 
Genus Planocarina Kosakyan, Lahr, Mulot, Meisterfeld, Mitchell and Lara, 2016

Planocarina carinata (Archer, 1867) Kosakyan, Lahr, Mulot, Meisterfeld, Mitchell and Lara, 2016

Nomenclature:

Nebela carinata (Archer, 1867) Leidy, 1879

Distribution: Rila Mt. (Golemansky and Todorov 1993); Stara Planina Mt. (new data); Vitosha Mt. (Golemansky and Todorov 1985, Golemansky and Todorov 1990, Todorov 1993, Todorov and Golemansky 1995).

Notes: So far, all records for the species have been as $N$. carinata.

Genus Quadrulella (Cockerell, 1909) sensu Kosakyan, Lahr, Mulot, Meisterfeld, Mitchell and Lara, 2016

Quadrulella longicollis (Taranek, 1882)

Nomenclature:

Quadrulella symmetrica var. longicollis Taranek, 1882

Distribution: Pirin Mt. (new data); Rhodopes Mt. (Golemansky et al. 2006); Rila Mt. (Todorov and Golemansky 2000, Todorov 2004, Todorov 2005, new data); Stara Planina Mt. (new data); Vitosha Mt. (Golemansky 1965, Golemansky and Todorov 1985, Golemansky and Todorov 1990, Todorov 1993, Todorov and Golemansky 1995, Kosakyan et al. 2012, new data).

Notes: So far, all records for the species (except this one of Kosakyan et al. 2012) have been as the infrasubspecific taxon Q. symmetrica var. longicollis (Rhodopes Mt., Rila Mt., Vitosha Mt.).

Quadrulella symmetrica (Wallich, 1864) Cockerell, 1909

Nomenclature:

Difflugia proteiformis var. symmetrica Wallich, 1863

Difflugia pyriformis var. symmetrica Wallich, 1864

Difflugia symmetrica Wallich, 1864

Assulina assulata Ehrenberg, 1871

Assulina leptolepis Ehrenberg, 1871

Difflugia assulata Ehrenberg, 1871

Difflugia carolensis Ehrenberg, 1871 
Quadrula symmetrica Schulze, 1875

Nebela (Quadrulella) symmetrica Deflandre, 1936

Distribution: Pirin Mt. (Golemansky 1974, new data); Rhodopes Mt. (Pateff 1924, Golemansky et al. 2006); Rila Mt. (Pateff 1924, Golemansky and Todorov 1993, Todorov and Golemansky 2000, Todorov 2004, Todorov 2005, new data); Stara Planina Mt. (new data); Vitosha Mt. (Pateff 1924, Golemansky 1965, Golemansky and Todorov 1985, Golemansky and Todorov 1990, Todorov 1993, Todorov and Golemansky 1995, Kosakyan et al. 2012, new data).

Notes: Besides the nominal species, the infrasubspecific taxon Quadrulella symmetrica var. irregularis Wailes et Penard, 1911 has also been recorded (Rhodopes Mt., Rila Mt., Vitosha Mt.).

Quadrulella tubulata Gauthier-Lièvre, 1953

Distribution: Pirin Mt. (Golemansky 1974).

Quadrulella variabilis Kosakyan, Lahr, Mulot, Meisterfeld, Mitchell and Lara, 2016

Distribution: Pirin Mt. (new data); Rila Mt. (new data); Stara Planina Mt. (new data); Vitosha Mt. (new data).

Suborder Phryganellina Bovee, 1985

Family Phryganellidae Jung, 1942

Genus Phryganella Penard, 1902

Phryganella acropodia (Hertwig and Lesser, 1874) Hopkinson, 1909

Nomenclature:

Difflugia acropodia Hertwig and Lesser, 1874

Distribution: Pirin Mt. (new data); Rhodopes Mt. (Golemansky et al. 2006); Rila Mt. (Todorov 2004, Todorov 2005, new data); Stara Planina Mt. (new data); Vitosha Mt. (Golemansky and Todorov 1985, Golemansky and Todorov 1990, Todorov 1993, Todorov and Golemansky 1995, new data).

Phryganella hemisphaerica (Penard, 1890) Penard, 1902

Nomenclature:

Pseudodifflugia hemisphaerica Penard, 1890 
Difflugia globulosa Leidy, 1879 (in part)

Distribution: Pirin Mt. (Golemansky 1974, new data); Rhodopes Mt. (Golemansky 1968, Golemansky et al. 2006); Rila Mt. (Todorov and Golemansky 2000, Todorov 2004,Todorov 2005, new data); Stara Planina Mt. (new data); Vitosha Mt. (new data)

\section{Phryganella nidulus Penard, 1902}

Nomenclature:

Difflugia globulosa Leidy, 1879 (in part)

Distribution: Pirin Mt. (new data); Rhodopes Mt. (Pateff 1924, Golemansky et al. 2006); Rila Mt. (Todorov 2004, Todorov 2005, new data); Vitosha Mt. (Pateff 1924).

Phryganella paradoxa Penard, 1902

Distribution: Rhodopes Mt. (Golemansky et al. 2006); Stara Planina Mt. (new data); Vitosha Mt. (Golemansky and Todorov 1985, Golemansky and Todorov 1990, Todorov 1993, Todorov and Golemansky 1995, new data).

Family Cryptodifflugiidae Jung, 1942

Genus Cryptodifflugia Penard, 1890

\section{Cryptodifflugia compressa Penard, 1902}

Distribution: Pirin Mt. (new data); Rhodopes Mt. (Golemansky 1968, Golemansky et al. 2006); Rila Mt. (new data); Stara Planina Mt. (new data); Vitosha Mt. (Golemansky 1965, Golemansky and Todorov 1990, new data).

Cryptodifflugia oviformis Penard, 1890

Nomenclature:

Difflugiella oviformis Bonnet and Thomas, 1955

Distribution: Pirin Mt. (new data); Rhodopes Mt. (Golemansky 1968, Golemansky et al. 2006); Rila Mt. (Golemansky and Todorov 1993, Todorov and Golemansky 2000, Todorov 2004, new data); Stara Planina Mt. (new data); Vitosha Mt. (Pateff 1928, Golemansky and Todorov 1985, Golemansky and Todorov 1990, Todorov 1993, Todorov and Golemansky 1995, new data).

Notes: The species has been recorded both as nominal species and as synonym $D$. oviformis (Rila Mt., Vitosha Mt.), as well as infrasubspecific taxon Difflugiella oviformis var. fusca Bonnet and Thomas, 1955 (Rhodopes Mt., Rila Mt., Vitosha Mt.). 
Genus Wailesella Deflandre, 1928

Wailesella eboracensis (Wailes and Penard, 1911) Deflandre, 1928

Nomenclature:

Cryptodifflugia eboracensis Wailes and Penard, 1911

Distribution: Pirin Mt. (new data); Rhodopes Mt. (Golemansky et al. 2006); Rila Mt. (Golemansky and Todorov 1993, Todorov and Golemansky 2000, new data); Stara Planina Mt. (new data); Vitosha Mt. (Pateff 1928, Golemansky 1965, Golemansky and Todorov 1985, Golemansky and Todorov 1990, Todorov 1993, Todorov and Golemansky 1995, new data).

Notes: The species has been recorded both as nominal species and as synonym $C$. eboracensis (Vitosha Mt.).

\section{Order Arcellinida INCERTAE SEDIS}

Genus Argynnia Vucetich, 1974

Argynnia bipes (Carter, 1870) Murray, 1870

Nomenclature:

Difflugia bipes Carter, 1870

Nebela bipes Murray, 1870

Nebela bicornis West, 1905

Distribution: Rhodopes Mt. (Pateff 1928, Golemansky et al. 2006); Vitosha Mt. (Pateff 1928, Golemansky and Todorov 1990).

Notes: The species has been recorded as synonym N. bipes (Rhodopes Mt., Vitosha Mt.).

\section{Argynnia dentistoma (Penard, 1890)}

Nomenclature:

Nebela dentistoma Penard, 1890

Nebela crenulata Penard, 1893

Nebela collaris Leidy, 1879 (in part)

Distribution: Pirin Mt. (Golemansky 1974, new data); Rhodopes Mt. (Pateff 1924, Golemansky 1968, Golemansky et al. 2006); Rila Mt. (Pateff 1924, Golemansky and Todorov 1993, Todorov and Golemansky 2000, Todorov 2005, new data); Stara 
Planina Mt. (new data); Vitosha Mt. (Pateff 1924, Golemansky 1965, Golemansky and Todorov 1985, Golemansky and Todorov 1990, Todorov 1993, Todorov and Golemansky 1995, new data).

Notes: So far, all records for the species have been as synonyms $N$. dentistoma (Pirin Mt., Rhodopes Mt., Rila Mt., Vitosha Mt.), N. crenulata (Rhodopes Mt., Rila Mt., Vitosha Mt.), as well as infrasubspecific taxa Nebela dentistoma var. oblonga GauthierLièvre, 1853 (Rila Mt.).

\section{Argynnia vitraea (Penard, 1899)}

Nomenclature:

Nebela vitraea Penard, 1899

Distribution: Pirin Mt. (new data); Rhodopes Mt. (Golemansky et al. 2006); Rila Mt. (Todorov and Golemansky 2000, Todorov 2004, Todorov 2005, new data); Stara Planina Mt. (new data); Vitosha Mt. (Golemansky and Todorov 1985, Golemansky and Todorov 1990, Todorov 1993, Todorov and Golemansky 1995, new data).

Notes: The species has been recorded as synonym N. vitraea (Rhodopes Mt., Rila Mt., Vitosha Mt.).

Genus Awerintzewia Schouteden, 1906

Awerintzewia cyclostoma (Penard, 1902) Schouteden, 1906

Nomenclature:

Heleopera cyclostoma Penard, 1902

Distribution: Rhodopes Mt. (Pateff 1924, Golemansky et al. 2006).

Notes: The species has been recorded both as nominal species and as synonym $H$. cyclostoma (Rhodopes Mt.).

Genus Heleopera Leidy, 1879

\section{Heleopera petricola Leidy, 1879}

Distribution: Pirin Mt. (new data); Rhodopes Mt. (Pateff 1924, Golemansky et al. 2006); Rila Mt. (Golemansky and Todorov 1993, Todorov 2005, new data); Stara Planina Mt. (new data); Vitosha Mt. (Pateff 1924, Golemansky 1965, Golemansky and Todorov 1985, Golemansky and Todorov 1990, Todorov 1993, Todorov and Golemansky 1995, new data). 
Notes: Besides the nominal species, two infrasubspecific taxa Heleopera petricola var. amethystea Penard, 1899 (Rila Mt.) and Heleopera petricola var. major Deflandre, 1928 (Vitosha Mt.) have also been recorded.

\section{Heleopera rosea Penard, 1890}

Distribution: Pirin Mt. (Golemansky 1974, new data); Rhodopes Mt. (Golemansky 1968, Golemansky et al. 2006); Rila Mt. (Golemansky and Todorov 1993, Todorov and Golemansky 2000, Todorov 2005, new data); Stara Planina Mt. (new data); Vitosha Mt. (Pateff 1924, Golemansky 1965, Golemansky and Todorov 1985, Golemansky and Todorov 1990, Todorov 1993, Todorov and Golemansky 1995, new data).

\section{Heleopera sphagni (Leidy, 1874)}

Nomenclature:

Difflugia (Nebela) sphagni Leidy, 1874

Nebela sphagni Leidy, 1876

Nebela picta Leidy, 1879

Distribution: Rhodopes Mt. (Golemansky 1968, Golemansky et al. 2006); Rila Mt. (Golemansky and Todorov 1993).

\section{Heleopera sylvatica Penard, 1890}

Distribution: Rhodopes Mt. (Golemansky et al. 2006); Rila Mt. (Golemansky and Todorov 1993, Todorov 2005, new data); Vitosha Mt. (Golemansky 1965, Golemansky and Todorov 1990).

\section{Genus Lesquereusia Schlumberger, 1845}

\section{Lesquereusia epistomium Penard, 1902}

Nomenclature:

Lecquereusia jurassica var. epistomium Penard, 1893

Lecquereusia epistomium Penard, 1902

Distribution: Pirin Mt. (new data); Rhodopes Mt. (Pateff 1924, Golemansky 1968,Golemansky et al. 2006); Rila Mt. (Todorov and Golemansky 2000, Todorov 2005, new data); Stara Planina Mt. (new data); Vitosha Mt. (Pateff 1924, Golemansky 1965, Golemansky and Todorov 1985, Golemansky and Todorov 1990, Todorov 1993, Todorov and Golemansky 1995, new data). 


\section{Lesquereusia gibbosa Thomas and Gauthier-Lièvre, 1859}

Distribution: Pirin Mt. (new data); Rhodopes Mt. (Golemansky 1968, Golemansky et al. 2006); Rila Mt. (Todorov 2005, new data); Stara Planina Mt. (new data); Vitosha Mt. (Golemansky and Todorov 1990, Todorov 1993, Todorov and Golemansky 1995, new data).

\section{Lesquereusia modesta Rhumbler, 1896}

\section{Nomenclature:}

Difflugia spiralis Leidy, 1879 (in part)

Distribution: Pirin Mt. (new data); Rhodopes Mt. (Golemansky 1968, Golemansky et al. 2006); Rila Mt. (Todorov 2004, Todorov 2005, new data); Stara Planina Mt. (new data); Vitosha Mt. (Golemansky 1965, Golemansky and Todorov 1990, Todorov 1993, Todorov and Golemansky 1995, new data).

\section{Lesquereusia spiralis (Ehrenberg, 1840) Bütschli, 1880}

Nomenclature:

Difflugia spiralis Ehrenberg, 1840

Lecquereusia jurassica Schlumberger, 1845

Difflugia helix Cohn, 1853

Distribution: Pirin Mt. (new data); Rhodopes Mt. (Pateff 1924, Golemansky 1968, Golemansky et al. 2006); Rila Mt. (Todorov and Golemansky 2000, Todorov 2004, Todorov 2005, new data); Stara Planina Mt. (new data); Vitosha Mt. (Pateff 1924, Golemansky 1965, Golemansky and Todorov 1985, Golemansky and Todorov 1990, Todorov 1993, Todorov and Golemansky 1995, new data).

\section{Genus Pyxidicula Ehrenberg, 1838}

Pyxidicula patens (Claparede and Lachmann, 1858)

Nomenclature:

Arcella patens Claparede and Lachmann, 1858

Distribution: Rhodopes Mt. (Golemansky 1968, Golemansky et al. 2006). 
Subkingdom / Supergroup Rhizaria Cavalier-Smith, 2002

Phylum Stramenopiles Patterson, 1989, emend. Adl et al., 2005

Class Labyrinthomycetes Dick, 2001

Order Amphitremida (Poch, 1913) Gomma, Mitchell and Lara, 2013

Family Amphitremidae Poch, 1913

Genus Archerella Loeblich and Tappan, 1961

Archerella flavum (Archer, 1877) Loeblich and Tappan, 1961

Nomenclature:

Ditrema flavum Archer, 1877

Amphitrema flavum Archer, 1877

Distribution: Rhodopes Mt. (Golemansky 1968, Golemansky et al. 2006); Rila Mt. (new data); Stara Planina Mt. (new data); Vitosha Mt. (Pateff 1924, Golemansky 1965, Golemansky and Todorov 1985, Golemansky and Todorov 1990, Todorov 1993, Todorov and Golemansky 1995, new data).

Notes: The species has been recorded as synonym A. flavum (Rhodopes Mt., Vitosha Mt.).

Phylum Cercozoa Cavalier-Smith, 1998, emend. Adl et al., 2005

Subphylum Filosa Cavalier-Smith, 2003

Class Silicofilosea Adl et al., 2005, emend. Adl et al., 2012

Order Euglyphida Copeland, 1956, emend. Cavalier-Smith, 1997

Family Euglyphidae Wallich, 1864

Genus Euglypha Dujardin, 1841

Euglypha acanthophora (Ehrenberg, 1841) Perty, 1849

Nomenclature: 
Difflugia acanthophora Ehrenberg, 1841

Euglypha alveolata Dujardin, 1841 (in part)

Euglypha setigera Perty, 1852 (in part)

Difflugia setigera Ehrenberg, 1871

Difflugia Setigerella acanthophora Ehrenberg, 1871

Euglypha brachiata Penard, 1902 (non Leidy, 1879)

Euglypha alveolata var. gracilis Taránek, 1881 (in part)

Euglypha armata Wailes and Penard, 1911

Euglypha australica Playfair, 1918 (in part)

Distribution: Pirin Mt. (Golemansky 1974, new data); Rhodopes Mt. (Pateff 1924, Golemansky 1968, Golemansky et al. 2006); Rila Mt. (Todorov and Golemansky 2000, Todorov 2004, Todorov 2005, new data); Stara Planina Mt. (new data); Vitosha Mt. (Pateff 1924, Golemansky 1965, Golemansky and Todorov 1985, Golemansky and Todorov 1990, Todorov 1993, Todorov and Golemansky 1995, new data).

Notes: Pateff (1924) and Golemansky et al. (2006) erroneously recorded the species E. brachiata (Rhodopes Mt., Vitosha Mt.), because the description of the found individuals fully corresponds to $E$. acanthophora.

\section{Euglypha aspera Penard, 1899}

Distribution: Pirin Mt. (new data); Rhodopes Mt. (Pateff 1924, Golemansky et al. 2006); Rila Mt. (Todorov 2004, new data).

\section{Euglypha bryophila Brown, 1911}

Nomenclature:

Euglypha $\alpha$ Vejdovsky, 1882

Euglypha cristata Penard, 1890 (in part)

Distribution: Pirin Mt. (new data); Rhodopes Mt. (Golemansky et al. 2006); Rila Mt. (Golemansky and Todorov 1993, Todorov and Golemansky 2000, new data); Stara Planina Mt. (new data); Vitosha Mt. (Golemansky 1965, Golemansky and Todorov 1990, new data).

Euglypha ciliata (Ehrenberg, 1848), Leidy, 1878

Nomenclature:

Difflugia ciliata Ehrenberg, 1848

Euglypha setigera Perty, 1852 (in part) 
Difflugia pilosa Ehrenberg, 1871

Difflugia Setigerella ciliata Ehrenberg, 1871

Difflugia Setigerella pilosa Ehrenberg, 1871

Distribution: Pirin Mt. (new data); Rhodopes Mt. (Pateff 1924, Golemansky 1968, Golemansky et al. 2006); Rila Mt. (Golemansky and Todorov 1993, Todorov and Golemansky 2000, Todorov 2004, Todorov 2005, new data); Stara Planina Mt. (new data); Vitosha Mt. (Pateff 1924, Golemansky and Todorov 1985, Golemansky and Todorov 1990, Todorov 1993, Todorov and Golemansky 1995, new data).

Notes: The species has been recorded both as nominal species and as infrasubspecific taxon E. ciliata f. glabra Wailes, 1915 (Rhodopes Mt., Rila Mt.).

\section{Euglypha compressa Carter, 1864}

Nomenclature:

Euglypha ampullacea Hertwig and Lesser, 1874

Euglypha ciliata Leidy, 1879 (in part)

Euglypha $\alpha$ Vejdovsky, 1882 (in part)

? Euglypha zonata Maggi, 1888

Distribution: Pirin Mt. (new data); Rhodopes Mt. (Golemansky et al. 2006); Rila Mt. (Todorov 2005, new data); Stara Planina Mt. (new data); Vitosha Mt. (Golemansky 1965, Golemansky and Todorov 1985, Golemansky and Todorov 1990, Todorov 1993, Todorov and Golemansky 1995, new data).

Notes: The species has been recorded both as nominal species and as infrasubspecific taxon E. compressa f. glabra Wailes, 1915 (Rhodopes Mt.).

\section{Euglypha crenulata Wailes, 1912}

Distribution: Vitosha Mt. (Golemansky and Todorov 1985, Golemansky and Todorov 1990, Todorov 1993, Todorov and Golemansky 1995).

Notes: The species has been recorded as infrasubspecific taxon E. crenulata var. minor Wailes, 1912.

\section{Euglypha cristata Leidy, 1874}

Distribution: Pirin Mt. (new data); Rhodopes Mt. (Golemansky 1968, Golemansky et al. 2006); Rila Mt. (Pateff 1924, Golemansky and Todorov 1993, Todorov and Golemansky 2000, Todorov 2005, new data); Stara Planina Mt. (new data); Vitosha Mt. (Pateff 1924, Golemansky 1965, Golemansky and Todorov 1985, Golemansky and Todorov 1990, Todorov 1993, Todorov and Golemansky 1995, new data). 


\section{Euglypha denticulata Brown, 1912}

Distribution: Pirin Mt. (new data); Rhodopes Mt. (Golemansky et al. 2006); Rila Mt. (Todorov and Golemansky 2000, Todorov 2005, new data); Stara Planina Mt. (new data); Vitosha Mt. (new data).

Euglypha filifera Penard, 1890

Nomenclature:

Euglypha setigera Perty, 1852 (in part)

Euglypha ciliata Leidy, 1879 (in part)

Euglypha longispina Taránek, 1881

Distribution: Pirin Mt. (Golemansky 1974, new data); Rhodopes Mt. (Golemansky et al. 2006); Rila Mt. (Todorov and Golemansky 2000, Todorov 2004, Todorov 2005, new data); Stara Planina Mt. (new data); Vitosha Mt. (Golemansky 1965, Golemansky and Todorov 1985, Golemansky and Todorov 1990,Todorov 1993, Todorov and Golemansky 1995, new data).

Euglypha laevis (Ehrenberg, 1845) Perty, 1849

Nomenclature:

Difflugia laevis Ehrenberg, 1845

Euglypha alveolata Leidy, 1879 (in part)

Euglypha $y$ Vejdovsky, 1882

Distribution: Pirin Mt. (Golemansky 1974, new data); Rhodopes Mt. (Golemansky 1968, Golemansky et al. 2006); Rila Mt. (Pateff 1924,Golemansky and Todorov 1993, Todorov and Golemansky 2000, Todorov 2005, new data); Stara Planina Mt. (new data); Vitosha Mt. (Golemansky 1965, Golemansky and Todorov 1985, Golemansky and Todorov 1990, Todorov 1993, Todorov and Golemansky 1995, new data).

\section{Euglypha rotunda Wailes and Penard, 1911}

Distribution: Pirin Mt. (new data); Rhodopes Mt. (Golemansky 1968, Golemansky et al. 2006); Rila Mt. (Golemansky and Todorov 1993, Todorov and Golemansky 2000, Todorov 2005, new data); Stara Planina Mt. (new data); Vitosha Mt. (Golemansky 1965, Golemansky and Todorov 1990, Todorov 1993, Todorov and Golemansky 1995, new data). 


\section{Euglypha strigosa (Ehrenberg, 1871) Leidy, 1878}

Nomenclature:

Difflugia strigosa Ehrenberg, 1871

Difflugia Setigerella strigosa Ehrenberg, 1871

Euglypha ciliata var. strigosa Leidy, 1879 (in part)

Euglypha heterospina Penard, 1890

Distribution: Pirin Mt. (Golemansky 1974, new data); Rhodopes Mt. (Golemansky 1968, Golemansky et al. 2006); Rila Mt. (Golemansky and Todorov 1993, Todorov and Golemansky 2000, Todorov 2005, new data); Stara Planina Mt. (new data); Vitosha Mt. (Pateff 1928, Golemansky 1965, Golemansky and Todorov 1985, Golemansky and Todorov 1990,Todorov 1993, Todorov and Golemansky 1995, new data).

Notes: Besides the nominal species, two infrasubspecific taxa Euglypha strigosa $\mathrm{f}$. glabra Wailes and Penard, 1911 (Rila Mt.) and Euglypha strigosa f. heterospina Wailes and Penard, 1911 (Rhodopes Mt., Rila Mt., Vitosha Mt.) have also been recorded.

\section{Euglypha tuberculata Dujardin, 1841}

Nomenclature:

Difflugia areolata Ehrenberg, 1841

Euglypha alveolata Dujardin, 1841 (in part)

Euglypha tuberculosa Dujardin, 1841

Difflugia alveolata Pritchard, 1861

Euglypha pusilla Entz, 1877

Euglypha $\beta$ Vejdovsky, 1882

Distribution: Pirin Mt. (Golemansky 1974, new data); Rhodopes Mt. (Pateff 1924, Golemansky et al. 2006); Rila Mt. (Golemansky and Todorov 1993, Todorov and Golemansky 2000, Todorov 2005, new data); Stara Planina Mt. (new data); Vitosha Mt. (Pateff 1924, Golemansky 1965, Golemansky and Todorov 1985, Golemansky and Todorov 1990, Todorov 1993, Todorov and Golemansky 1995, new data).

Notes: The species has been recorded both as nominal species and as synonym $E$. alveolata (Rhodopes Mt., Vitosha Mt.). 
Family Trinematidae Hoogenraad and De Groot 1940, emend Adl et al. 2012

Genus Trinema Dujardin, 1841

Trinema complanatum Penard, 1890

Nomenclature:

? Arcella nidus-pendulus Ehrenberg, 1841

Trinema acinus Leidy, 1879 (in part)

Distribution: Pirin Mt. (new data); Rhodopes Mt. (Pateff 1924, Golemansky 1968, Golemansky et al. 2006); Rila Mt. (Golemansky and Todorov 1993, Todorov and Golemansky 2000, Todorov 2005, new data); Stara Planina Mt. (new data); Vitosha Mt. (Pateff 1924, Golemansky 1965, Golemansky and Todorov 1985, Golemansky and Todorov 1990, Todorov 1993, Todorov and Golemansky 1995, new data).

Notes: Besides the nominal species, two infrasubspecific taxa Trinema complanatum var. aerophila (Decloître, 1950) Bonnet and Thomas, 1960 (Vitosha Mt.) and Trinema complanatum var. globulosa Chardez, 1959 (Rhodopes Mt., Vitosha Mt.) have also been recorded.

Trinema enchelys (Ehrenberg, 1838) Leidy, 1878

Nomenclature:

Difflugia enchelys Ehrenberg, 1838 (in part)

Trinema acinus Dujardin, 1841

Arcella enchelys Ehrenberg, 1844

Euglypha pleurostoma Carter, 1857

Euglypha enchelys Wallich, 1864

Trinema (Difflugia) encheli Crevier, 1870

Distribution: Pirin Mt. (Golemansky 1974, new data); Rhodopes Mt. (Pateff 1924, Golemansky 1968, Golemansky et al. 2006); Rila Mt. (Pateff 1924, Golemansky and Todorov 1993, Todorov and Golemansky 2000, Todorov 2005, new data); Stara Planina Mt. (new data); Vitosha Mt. (Pateff 1924, Golemansky 1965, Golemansky and Todorov 1985, Golemansky and Todorov 1990, Todorov 1993, Todorov and Golemansky 1995, new data).

Trinema galeata (Penard, 1890) Jung, 1942

Nomenclature:

Trinema enchelys var. galeata Penard, 1890 
Distribution: Rila Mt. (Golemansky and Todorov 1993).

Trinema grandis (Chardez, 1960) Golemansky, 1963

Nomenclature:

Trinema enchelys var. grandis Chardez, 1960

Distribution: Rila Mt. (Golemansky and Todorov 1993, new data).

Trinema lineare Penard, 1890

Nomenclature:

Difflugia enchelys Ehrenberg, 1838 (in part)

Arcella hyalina Ehrenberg, 1841

Arcella enchelys Ehrenberg, 1854

Trinema acinus Leidy, 1879 (in part)

Trinema enchelys forma $\beta$ Awerintzew, 1906

Distribution: Pirin Mt. (Golemansky 1974, new data); Rhodopes Mt. (Golemansky 1968, Golemansky et al. 2006); Rila Mt. (Golemansky and Todorov 1993, Todorov and Golemansky 2000, Todorov 2004, Todorov 2005, new data); Stara Planina Mt. (new data); Vitosha Mt. (Golemansky 1965, Golemansky and Todorov 1985, Golemansky and Todorov 1990, Todorov 1993, Todorov and Golemansky 1995, new data).

\section{Genus Corythion Taranek, 1881}

Corythion delamarei Bonnet and Thomas, 1960

Distribution: Vitosha Mt. (Todorov 1993, Todorov and Golemansky 1995).

\section{Corythion dubium Taránek, 1881}

Nomenclature:

Arcella constricta Ehrenberg, 1841 (in part)

Arcella disphaera Ehrenberg, 1841 (in part)

Trinema acinus Leidy, 1879 (in part)

Trinema constricta Certes, 1889

Distribution: Pirin Mt. (Golemansky 1974, new data); Rhodopes Mt. (Golemansky 1968, Golemansky et al. 2006); Rila Mt. (Pateff 1924, Golemansky and Todorov 1993, Todorov and Golemansky 2000, Todorov 2004, Todorov 2005, new data); Stara Planina Mt. (new data); Vitosha Mt. (Pateff 1924, Golemansky 1965, Golemansky and 
Todorov 1985, Golemansky and Todorov 1990, Todorov 1993, Todorov and Golemansky 1995, new data).

Notes: Besides the nominal species, two infrasubspecific taxa Corythion dubium var. aerophila Decloître, 1950 (Vitosha Mt.) and Corythion dubium var. orbicularis Penard, 1910 (Vitosha Mt.) have also been recorded.

Genus Playfairina Thomas, 1961

\section{Playfairina valkanovi Golemansky, 1966}

Distribution: Pirin Mt. (new data); Rhodopes Mt. (Golemansky et al. 2006); Rila Mt. (Golemansky and Todorov 1993, Todorov and Golemansky 2000, Todorov 2005, new data); Stara Planina Mt. (new data); Vitosha Mt. (Golemansky 1966, Golemansky and Todorov 1990, Todorov 1993, Todorov and Golemansky 1995, Golemansky and Todorov 2006, new data).

Family Sphenoderiidae Chatelain, Meisterfeld, Roussel-Delif and Lara, 2013

Genus Sphenoderia Schlumberger, 1845

Sphenoderia fissirostris Penard, 1890

Distribution: Pirin Mt. (Golemansky 1974, new data); Rhodopes Mt. (Golemansky 1968, Golemansky et al. 2006); Rila Mt. (Pateff 1924, Golemansky and Todorov 1993, Todorov and Golemansky 2000, Todorov 2004, Todorov 2005, new data); Stara Planina Mt. (new data); Vitosha Mt. (Pateff 1924, Golemansky 1965, Golemansky and Todorov 1985, Golemansky and Todorov 1990, Todorov 1993,Todorov and Golemansky 1995, new data).

\section{Sphenoderia lenta Schlumberger, 1845}

Distribution: Pirin Mt. (Golemansky 1974, new data); Rhodopes Mt. (Pateff 1924, Golemansky et al. 2006); Rila Mt. (Pateff 1924, Todorov and Golemansky 2000, Todorov 2004, Todorov 2005, new data); Stara Planina Mt. (new data); Vitosha Mt. (Pateff 1924, Golemansky 1965, Golemansky and Todorov 1985, Golemansky and Todorov 1990, Todorov 1993, Todorov and Golemansky 1995, new data).

\section{Sphenoderia minuta Deflandre, 1931}

Distribution: Pirin Mt. (new data); Rhodopes Mt. (Golemansky 1968, Golemansky et al. 2006); Rila Mt. (Golemansky and Todorov 1993, new data); Stara Planina Mt. (new data); Vitosha Mt. (Golemansky 1965, Golemansky and Todorov 1990, Todorov 1993, Todorov and Golemansky 1995, new data). 
Sphenoderia ovoidea Jung, 1942

Distribution: Stara Planina Mt. (new data); Vitosha Mt. (new data).

Sphenoderia splendida (Playfair, 1917) Deflandre, 1931

Nomenclature:

Sphenoderia fissirostris var. splendida Playfair, 1917

Distribution: Pirin Mt. (new data); Rila Mt. (new data); Stara Planina Mt. (new data); Vitosha Mt. (new data).

Genus Trachelocorythion Bonnet, 1979

Trachelocorythion pulchellum (Penard, 1890) Bonnet, 1979

Nomenclature:

? Euglypha minima Perty, 1852

Corythion pulchellum Penard, 1890

Chorythion pulchellum Awerintzew, 1907

? Hyalina neta Jung, 1942

Distribution: Pirin Mt. (new data); Rhodopes Mt. (Golemansky et al. 2006); Rila Mt. (Todorov and Golemansky 2000, new data); Stara Planina Mt. (new data); Vitosha Mt. (new data).

Family Assulinidae Lara et al., 2007

Genus Assulina Leidy, 1879

\section{Assulina muscorum Greeff, 1888}

Nomenclature:

Assulina seminulum Leidy, 1879 (in part)

Assulina minor Penard, 1890

Distribution: Pirin Mt. (new data); Rhodopes Mt. (Golemansky 1968, Golemansky et al. 2006); Rila Mt. (Golemansky and Todorov 1993, Todorov and Golemansky 2000, Todorov 2005, new data); Stara Planina Mt. (new data); Vitosha Mt. (Golemansky 1965, Golemansky and Todorov 1990, Todorov 1993, Todorov and Golemansky 1995, new data). 
Assulina seminulum (Ehrenberg, 1848) Leidy, 1879

Nomenclature:

Difflugia seminulum Ehrenberg, 1848

Difflugia Assulina seminulum Ehrenberg, 1871

Difflugia semen Ehrenberg, 1871

Euglypha brunnea Leidy, 1874

Euglypha seminulum Leidy, 1878

Distribution: Rhodopes Mt. (Golemansky 1968, Golemansky et al. 2006); Rila Mt. (Pateff 1924, Golemansky and Todorov 1993, new data); Stara Planina Mt. (new data); Vitosha Mt. (Pateff 1924, Golemansky 1965, Golemansky and Todorov 1990, new data).

Genus Placocista Leidy, 1879

Placocista glabra Penard, 1906

Distribution: Pirin Mt. (Golemansky 1974).

Notes: The species has been recorded as infrasubspecific taxon Placocista glabra var. minima Decloître, 1955 (Pirin Mt.).

Placocista spinosa (Carter, 1865) Leidy, 1879

Nomenclature:

Euglypha spinosa Carter, 1865

Distribution: Rila Mt. (Golemansky and Todorov 1993).

Family Cyphoderiidae de Saedeleer, 1934

Genus Cyphoderia Schlumberger, 1845

Cyphoderia amphoralis (Wailes and Penard, 1911)

Nomenclature:

Cyphoderia trochus var. amphoralis Wailes and Penard, 1911

Distribution: Pirin Mt. (new data); Rhodopes Mt. (Todorov et al. 2009); Rila Mt. (Heger et al. 2010, new data); Stara Planina Mt. (new data); Vitosha Mt. (new data). 


\section{Cyphoderia ampulla (Ehrenberg, 1840) Leidy, 1878}

Nomenclature:

Difflugia ampulla Ehrenberg, 1840

Difflugia lagena Ehrenberg, 1841

Cyphoderia margaritacea Schlumberger, 1845

Euglypha curvata Perty, 1852

Lagynis baltica Schultze, 1854

Euglypha ampulla Claparède and Lachmann, 1859

Euglypha baltica Wallich, 1864

Euglypha margaritacea Wallich, 1864

Difflugia Seelandica Ehrenberg, 1869

Difflugia adunca Ehrenberg, 1871

Difflugia alabamensis Ehrenberg, 1871

Difflugia uncinnata Ehrenberg, 1871

Difflugia margaritacea Ehrenberg, 1871

Distribution: Pirin Mt. (Golemansky 1974, new data); Rhodopes Mt. (Pateff 1924, Golemansky et al. 2006; Todorov et al. 2009, Heger et al. 2010); Rila Mt. (Golemansky and Todorov 1993, Todorov and Golemansky 2000, Todorov 2004, Todorov 2005, Todorov et al. 2009, new data); Stara Planina Mt. (new data); Vitosha Mt. (Pateff 1924, Golemansky 1965, Golemansky and Todorov 1985, Golemansky and Todorov 1990, Todorov 1993, Todorov and Golemansky 1995, Todorov et al. 2009, Heger et al. 2010, new data).

\section{Cyphoderia major (Penard, 1891)}

Nomenclature:

Cyphoderia margaritacea var. major Penard, 1891

Distribution: Pirin Mt. (new data); Rila Mt. (Todorov et al. 2009, Heger et al. 2010, new data); Stara Planina Mt. (new data); Vitosha Mt. (new data).

\section{Genus Campascus Leidy, 1879}

\section{Campascus minutus Penard, 1899}

Nomenclature:

Campascus triqueter var. minuta Awerintzew, 1906 
Distribution: Rhodopes Mt. (Golemansky et al. 2006); Rila Mt. (Todorov and Golemansky 2000, new data); Stara Planina Mt. (new data); Vitosha Mt. (Golemansky 1965, Golemansky and Todorov 1990, new data).

\section{Campascus triqueter Penard, 1891}

Distribution: Rila Mt. (Golemansky and Todorov 1993, Todorov and Golemansky 2000); Vitosha Mt. (Golemansky 1965, Golemansky and Todorov 1990, Todorov 1993, Todorov and Golemansky 1995).

Family Paulinellidae de Saedeller 1934, emend. Adl et al. 2012

Genus Paulinella Lauterborn, 1895

Paulinella chromatophora Lauterborn, 1895

Distribution: Rhodopes Mt. (Golemansky et al. 2006); Rila Mt. (Todorov 2004).

Order INCERTAE SEDIS euglyphid testate amoebae

Genus Tracheleuglypa Deflandre, 1928

Tracheleuglypa acolla Bonnet et Thomas, 1955

Distribution: Pirin Mt. (new data); Rhodopes Mt. (Golemansky et al. 2006); Rila Mt. (Todorov and Golemansky 2000, Todorov 2005, new data); Stara Planina Mt. (new data); Vitosha Mt. (Todorov 1993, Todorov and Golemansky 1995, new data).

Tracheleuglypa dentata (Moniez, 1888) Deflandre, 1928

Nomenclature:

Sphenoderia lenta Leidy, 1879 (in part)

Euglypha $\beta$ Vejdovsky, 1882

Euglypha dentata Moniez, 1888

Sphenoderia dentata Penard, 1890

Distribution: Pirin Mt. (Golemansky 1974, new data); Rhodopes Mt. (Golemansky 1968, Golemansky et al. 2006); Rila Mt. (Golemansky and Todorov 1993, Todorov and Golemansky 2000, Todorov 2005 new data); Stara Planina Mt. (new data); Vitosha Mt. (Pateff 1924, Golemansky 1965, Golemansky and Todorov 1985, Golemansky and Todorov 1990, Todorov 1993, Todorov and Golemansky 1995, new data). 
Notes: The species has been recorded both as nominal species and as synonym $S$. dentata (Vitosha Mt.).

Family Chlamydophryidae de Saedeleer, 1934

Genus Lecythium Hertwig and Lesser, 1874

Lecythium mutabilis (Bailey, 1853)

Nomenclature:

Pamphagus mutabilis Bailey, 1853

Pamphagus avidus Leidy, 1879

Lecythium mutabile Wailes, 1915

Distribution: Rila Mt. (Pateff 1924).

Notes: The species has been recorded as synonym P. mutabilis (Rila Mt.).

Family Pseudodifflugiidae de Saedeleer, 1934

Genus Pseudodifflugia Schlumberger, 1845

Pseudodifflugia fascicularis Penard, 1902

Distribution: Rhodopes Mt. (Golemansky 1968, Golemansky et al. 2006); Rila Mt. (Pateff 1924).

\section{Pseudodifflugia gracilis Schlumberger, 1845}

Nomenclature:

Pleurophrys sphaerica Claparède and Lachmann, 1858

Pleurophrys angulata Mereschkovsky, 1879

Distribution: Rhodopes Mt. (Golemansky et al. 2006); Rila Mt. (Golemansky and Todorov 1993, Todorov and Golemansky 2000).

\section{Discussion}

Prior to our investigation, the number of known Sphagnum-dwelling testate amoebae in Bulgaria was 155. Our study increases this number with 16 species and the present checklist comprises 171 species classified into 43 genera, 20 families, three orders, three classes and three phyla. We present data for 16 new Sphagnum-dwelling testate amoebae 
in Bulgaria and new distribution data for 134 species. Of them, 99 species are recorded from Stara Planina Mt., for which there was no available data to date. Additionally we report 69 new species for Pirin Mt., 21 for Vitosha Mt. and 18 for Rila Mt. Thirty six species have been synonymised according to the latest taxonomic changes. All known synonyms of valid names are also listed. The species Euglypha brachiata Penard, 1902 and the infrasubspecific taxa Difflugia compressa var. africana Gauthier-Lièvre et Thomas, 1958 have erroneously been recorded, because the descriptions of the found individuals fully correspond to $E$. acanthophora and $Z$. compressa, respectively. These misidentified taxa have been transferred to valid species. Three of the recorded species have not been included in the checklist, because they are currently not refering to testate amoebae ( Cochliopodium bilimbosum and $C$. echinatum are naked amoebae and Microgromia elegantula is freshwater foraminifera).

About $30 \%$ (51 species) of all recorded testate amoebae are typical sphagnicolous inhabitants, which only exceptionally can be found in other habitats. These are mostly the representatives of the genera Nebela (5 species), Sphenoderia (5), Lesquereusia (4), Longinebela (4), Padaungiella (4), Quadrulella (4), Heleopera (3) etc. The most numerous (67 species, 39\%) are the species which are found in Sphagnum mosses but are also common inhabitants of freshwater basins. The genera Difflugia (28), Centropyxis (7) and Arcella (6) have the largest number of representatives in this group. Most likely, some of these species are not usual inhabitants and fall incidentally into the Sphagnum mosses, which develop around the shores of freshwater basins (e.g. Difflugia lobostoma, D. microclaviformis, D.urceolata, Netzelia tuberculata etc.). The relative share of species that are rather inhabitants of soils and soil mosses, but also occur in peatlands is comparatively smaller (about 18\%, 31 species). Most of them rarely occur and usually have a low population density in Sphagnum mosses (e.g. Awerintzewia cyclostoma, Bullinularia indica, Centropyxis cryptostoma, Centropyxis orbicularis, Cyclopyxis arceloides, Cyclopyxis puteus , Heleopera sylvatica, Padaungiella wailesi, Plagiopyxis callida, Plagiopyxis labiata, Trinema galeata etc.). The number of eurybiotic species, which are widely distributed in diverse habitats, including peatlands, is the smallest. Twenty one species (about $12 \%$ of all recorded) can be referred to eurybionts. Of these, the most frequently occurring in Spagnum mosses are Centropyxis aerophila, Corythion dubium, Cyclopyxis eurystoma, Euglypha laevis, Phryganella acropodia, Trinema lineare etc.

On a generic level, the genera Difflugia (32), Centropyxis (17), Euglypha (13), Arcella (10) and Nebela (8) have the greatest species richness. These five genera constitute about $47 \%$ of all testate amoebae recorded in Sphagnum mosses in Bulgaria. Of the other genera, 23 are represented with 2-5 species and 15 with one species only.

From families, the largest richness have Difflugiidae (39 species, 4 genera), Hyalospheniidae $(26,7)$, Centropyxidae $(17,1)$, Euglyphidae $(13,1)$ and Arcellidae $(10,1)$, which together make up two thirds of Sphagnum-dwelling testate amoebae in Bulgaria. Six families are represented by one species of one genus only (Microcoyciidae, Microchlamyidae, Netzeliidae, Amphitremidae, Paulinellidae and Chlamydophryidae). 
At a higher taxonomic level, the most numerous is the order Arcellinida, which includes 128 species of 28 genera and 11 families, followed by the order Euglyphida with 42 species of 14 genera and 8 families and finally by the order Amphitremida with one species of 1 genus and 1 family only.

\section{Acknowledgements}

This work was supported by a grant from the Bulgarian Academy of Sciences, Program for career development of young scientists (grant DFNP-17-51/26.07.2017 to Nikola Bankov). The scanning electron microscope study was supported by the National Endowment Fund "13 Centuries Bulgaria". We thank the reviewer Prof. Edward Mitchell (University of Neuchatel) for his helpful and constructive comments and editorial suggestions, which enchanced the quality of the manuscript. The authors wish to acknowledge Nikola Dimitrov (Faculty of Chemistry and Pharmacy, Sofia University) for his help and advice with the scanning electron microscopy.

\section{References}

- $\quad$ Adl SM, Simpson AGB, Lane CE, Lukeš J, Bass D, Bowser SS, Brown MW, Burki F, Dunthorn M, Hampl V, Heiss A, Hoppenrath M, Lara E, le Gall L, Lynn DH, McManus H, Mitchell EAD, Mozley-Stanridge SE, Parfrey LW, Pawlowski J, Rueckert S, Shadwick L, Schoch CL, Smirnov A, Spiegel FW (2012) The revised classification of eukaryotes. Journal of Eukaryotic Microbiology 59: 429-514. https://doi.org/10.1111/ j.1550-7408.2012.00644.x

- Aoki, Yoshiyuki, Hoshino, Mitsuo, Matsubara, Teruo (2007) Silica and testate amoebae in a soil under pine-oak forest. Geoderma 142: 29-35. https://doi.org/10.1016/ j.geoderma.2007.07.009

- $\quad$ Blandenier Q, Lara E, Mitchell EAD, Alcantara DMC, Siemensma FJ, Todorov M, Lahr DJG (2017) NAD9/NAD7 (mitochondrial nicotinamide adenine dinucleotide dehydrogenase gene)—A new "Holy Grail" phylogenetic and DNA-barcoding marker for Arcellinida (Amoebozoa)? European Journal of Protistology 58: 175-186. https:// doi.org/10.1016/i.ejop.2016.12.002

- Booth RK (2002) Testate amoebae as paleoindicators of surface-moisture changes on Michigan peatlands: Modern ecology and hydrological calibration. Journal of Paleolimnology 28 (3): 329-348. https://doi.org/10.1023/A:1021675225099

- Charman DJ, Roe HM, Gehrels WR (1998) The use of testate amoebae in studies of sea-level change: a case study from the Taf Estuary, south Wales, UK. The Holocene 8 (2): 209-218. https://doi.org/10.1191/095968398676389446

- Charman DJ (2001) Biostratigraphic and palaeoenvironmental applications of testate amoebae. Quaternary Science Reviews 20: 1753-1764. https://doi.org/10.1016/ s0277-3791(01)00036-1

- Chatelain AP, Meisterfeld R, Roussel-Delif L, Lara E (2013) Sphenoderiidae (fam. nov.), a new clade of euglyphid testate amoebae characterized by small, round scales 
surrounding the aperture. Protist 164 (6): 782-792. https://doi.org/10.1016/ j.protis.2013.08.001

- Golemansky V (1965) Étude sur les espèces et l'écologie des rhizopodes (Rhizopoda, Testacea) des hautes tourbières de la montagne Vitocha. Annuaire de l'Université de Sofia, Faculté de Biologie. 59 (1): 17-38. [In Bulgarian, with Russian and French summaries].

- Golemansky V (1966) Playfairina valkanovi nov. sp. - une nouvelle rhizopode d'eau douce (Rhizopoda, Testacea). Comptes Rendus de l'Académie Bulgare des Sciences 19 (1): 57-59.

- Golemansky V (1968) On the species composition and distribution of the rhizopod fauna of upper Smolian Lakes. Bulletin de l'Institut de Zoologie et Musée de Sofia 27: 171-187. [In Bulgarian, with Russian and English summaries].

- $\quad$ Golemansky V (1974) La faune rhizopodique (Rhizopoda, Testacea) du littoral et du benthal du lac Popovo dans la montagne de Pirine. Bulletin de l'Institut de Zoologie et Mesée 40: 47-58. [In Bulgarian, with Russian and French summaries].

- Golemansky V, Todorov M (1985) Comparative studies on the composition and distribution of the thecamoeban fauna (Rhizopoda, Testacea) in three types of soil in Vitosha Mountain. Acta Zoologica Bulgarica 29: 50-64. [In Bulgarian, with Russian and English summaries].

- $\quad$ Golemansky V, Todorov M (1990) Rhizopodic fauna (Protozoa, Rhizopoda) from Vitoša. In: Fauna of Southwestern Bulgaria, Part 3. BAS, Sofia, 19-48. [In Bulgarian, with Russian and English summaries].

- $\quad$ Golemansky V, Todorov M (1993) Testate amoebae (Protozoa, Rhizopoda) in the watercatchment area and littoral of the "Beli Iskar" dam. Acta Zoologica Bulgarica 46: 3-9.

- Golemansky V, Todorov M (2006) Morphology, biometry and ecology of Playfairina valkanovi Golemansky, 1966 (Testaceafilosia: Trinematidae), with a supplement to the diagnosis of the genus Playfairina Thomas, 1961. Acta Zoologica Bulgarica 58 (3): 291-298.

- Golemansky V, Todorov M, Temelkov B (2006) Diversity and biotopic distribution of the Rhizopods (Rhizopoda: Lobosia and Filosia) from the Western Rhodopes (Bulgaria). In: Beron, P. Biodiversity of Bulgaria. 3. Biodiversity of Western Rhodopes (Bulgaria and Greece) I. Pensoft \& National Museumof Natural Histoty, Sofia, 205-220 pp.

- Gomaa F, Mitchell EAD, Lara E (2013) Amphitremida (Poche, 1913) is a new major, ubiquitous labyrinthulomycete clade. PLoS ONE 8 (1): e53046. https://doi.org/10.1371/ journal.pone.0053046

- Gomaa F, Lahr DJ, Todorov M, Li J, Lara E (2017) A contribution to the phylogeny of agglutinating Arcellinida (Amoebozoa) based on SSU rRNA gene sequences. European Journal of Protistology 59: 99-107. https://doi.org/10.1016/..ejop.2017.03.005

- Heger TJ, Mitchell EAD, Todorov M, Golemansky V, Lara E, Leander BS, Pawlowski J (2010) Molecular phylogeny of euglyphid testate amoebae (Cercozoa: Euglyphida) suggests transitions between marine supralittoral and freshwater/terrestrial environments are infrequent. Molecular Phylogenetics and Evolution 55: 113-122. https://doi.org/10.1016/i.ympev.2009.11.023

- Kosakyan A, Heger TJ, Leander BS, Todorov M, Mitchell EA, Lara E (2012) COI barcoding of nebelid testate amoebae (Amoebozoa: Arcellinida): extensive cryptic 
diversity and redefinition of the Hyalospheniidae Schultze. Protist 163: 415-434. https:// doi.org/10.1016/j.protis.2011.10.003

- Kosakyan A, Gomaa F, Lara E, Lahr DJ (2016a) Current and future perspectives on the systematics, taxonomy and nomenclature of testate amoebae. European Journal of Protistology 55: 105-117. https://doi.org/10.1016/j.ejop.2016.02.001

- $\quad$ Kosakyan A, Lahr DJG, Mulot M, Meisterfeld R, Mitchell EAD, Lara E (2016b) Phylogenetic reconstruction based on $\mathrm{COI}$ reshuffles the taxonomy of hyalosphenid shelled (testate) amoebae and reveals the convoluted evolution of shell plate shapes. Cladistics 32 (6): 606-623. https://doi.org/10.1111/cla.12167

- Lahr DJ, Grant JR, Katz LA (2013) Multigene phylogenetic reconstruction of the Tubulinea (Amoebozoa) corroborates four of the six major lineages, while additionally revealing that shell composition does not predict phylogeny in the Arcellinida. Protist 164 (3): 323-339. https://doi.org/10.1016/i.protis.2013.02.003

- $\quad$ Lamentowicz M, Mitchell EAD (2005) The ecology of testate amoebae (Protists) in Sphagnum in north-western Poland in relation to peatland ecology. Microbial Ecology 50 (1): 48-63. https://doi.org/10.1007/s00248-004-0105-8

- Lara E, Heger TJ, Mitchell EAD, Meisterfeld R, Ekelund F (2007) SSU rRNA reveals a sequential increase in shell complexity among the euglyphid testate amoebae (Rhizaria: Euglyphida). Protist 158 (2): 229-237. https://doi.org/10.1016/i.protis.2006.11.006

- $\quad$ Mazei Y, Warren A (2012) A survey of the testate amoeba genus Difflugia Leclerc, 1815 based on specimens in the E. Penard and C.G. Ogden collections of the Natural History Museum, London. Part 1: species with shells that are pointed aborally and/or have aboral protuberances. Protistology 7 (3): 121-171.

- $\quad$ Mazei Y, Warren A (2014) A survey of the testate amoeba genus Difflugia Leclerc, 1815 based on specimens in the E. Penard and C.G. Ogden collections of the Natural History Museum, London. Part 2: species with shells that are pyriform or elongate. Protistology 8 (4): 133-171.

- $\quad$ Mazei Y, Warren A (2015) A survey of the testate amoeba genus Difflugia Leclerc, 1815 based on specimens in the E. Penard and C.G. Ogden collections of the Natural History Museum, London. Part 3: species with shells that are spherical or ovoid. Protistology 9 (1): 3-49.

- Meisterfeld R (2002a) Order Arcellinida Kent, 1880. In: Lee JJ, Leedale GE, Bradbury P (Eds) The illustrated guide to the Protozoa. Second edition, Volume II. Society of protozoologists, Lawrence, Kansas, USA, 827-860 pp. [ISBN 1-891276-23-9].

- Meisterfeld R (2002b) Testate amoebae with Filopodia. In: Lee JJ, Leedale GF, Bradbury P (Eds) The illustrated guide to the Protozoa . Second edition, Volume II. Society of protozoologists, Lawrence, Kansas, USA, 1054-1084 pp. [ISBN 1-891276-23-9].

- Mitchell EAD, Charman DJ, Warner BG (2008) Testate amoebae analysis in ecological and paleoecological studies of wetlands: past, present and future. Biodiversity and Conservation 17 (9): 2115-2137. https://doi.org/10.1007/s10531-007-9221-3

- Natcheva R, Ganeva A (2005) Check-list of the bryophytes of Bulgaria II. Musci . Cryptogamie Bryologie 26: 209-232.

- $\quad$ Nguyen-Viet H, Bernard N, Mitchell EAD, Cortet J, Badot PM, Gilbert D (2007) Relationship between testate amoeba (Protist) communities and atmospheric heavy metals accumulated in Barbula indica (Bryophyta) in Vietnam. Microbial Ecology 53 (1): 53-65. https://doi.org/10.1007/s00248-006-9108-y 
- $\quad$ Pateff P (1924) Kenntnis der Süsswasserrhizopoden von Bulgarien. Arbeiten der Bulgarischen Naturforschenden Gesellschaft 11: 1-46. [In Bulgarian, with German summary].

- $\quad$ Pateff $P$ (1928) Einige neue Süsswassershizopoden von Bulgarien. Arbeiten der Bulgarischen Naturforschenden Gesellschaft 13: 131-136. [In Bulgarian, with German summary].

- $\quad$ Qin Y, Mitchell EAD, Lamentowicz M, Payne RJ, Lara E, Gu Y, Huang X, Wang H (2013) Ecology of testate amoebae in peatlands of central China and development of a transfer function for paleohydrological reconstruction. Journal of Paleolimnology 50 (3): 319-330. https://doi.org/10.1007/s10933-013-9726-6

- Swindles GT, Charman DJ, Roe HM, Sansum PA (2008) Environmental controls on peatland testate amoebae (Protozoa: Rhizopoda) in the North of Ireland: Implications for Holocene palaeoclimate studies. Journal of Paleolimnology 42 (1): 123-140. https:// doi.org/10.1007/s10933-008-9266-7

- $\quad$ Swindles GT, Amesbury MJ, Turner TE, Carrivick JL, Woulds C, Raby C, Mullan D, Roland TP, Galloway JM, Parry L, Kokfelt U, Garneau M, Charman DJ, Holden J (2015) Evaluating the use of testate amoebae for palaeohydrological reconstruction in permafrost peatlands. Palaeogeography, Palaeoclimatology, Palaeoecology 424:

111-122. https://doi.org/10.1016/j.palaeo.2015.02.004

- Todorov M (1993) Testate Amoebae (Protozoa, Rhizopoda) in soils of Vitoša Mountain (Bulgaria). Acta Zoologica Bbulgarica 46: 16-23.

- Todorov M, Golemansky V (1995) Ecological caracteristics of soil Testacea (Protozoa, Rhizopoda) of Vitoša Mountain (Bulgaria). Acta Zoologica Bulgarica 48: 3-22.

- Todorov M, Golemansky V (2000) Testate Amoebae (Protozoa: Testacea) of the Glacial Lakes in the Rila National Park (Southwestern Bulgaria). In: Golemansky V, Naidenow W (Eds) Biodiversity and Evolution of Glacial Water Ecosystems in the Rila Mountains. Professor Marin Drinov Academic Publlshing House, Sofia, 15-26 pp.

- Todorov M (2004) Testate amoebae (Protozoa, Rhizopoda) of the Ribni Ezera Glacial Lakes in the Rila Mountains (South-West Bulgaria). Acta Zoologica Bulgarica 56 (3): 243-252.

- $\quad$ Todorov M (2005) Testate amoebae (Protozoa, Rhizopoda) of the glacial lakes Smradlivo ezero in the Rila National Park (Southwestern Bulgaria). Acta Zoologica Bulgarica 57 (1): 13-23.

- Todorov M, Golemansky V, Mitchell EAD, Heger TJ (2009) Morphology, biometry, and taxonomy of freshwater and marine interstitial Cyphoderia (Cercozoa: Euglyphida). Journal of Eukaryotic Microbiology 56 (3): 279-289. https://doi.org/10.1111/ j.1550-7408.2009.00394.x

- $\quad$ Todorov M (2010) Nebela golemanskyi sp. nov., a new sphagnicolous testate amoeba from Bulgaria (Amoebozoa: Arcellinida, Nebelidae). Acta Protozoologica 49 (1): 37-43.

- $\quad$ Todorov M, Golemansky V, Meisterfeld R (2010) Is Difflugia nebeloides (Amoebozoa: Arcellinida) really a Difflugia? Re-description and new combination. Acta Zoologica Bulgarica 62 (1): 13-20.

- $\quad$ Todorov M, Bankov N, Ganeva A (2018) Longinebela ampulla sp. nov. (Arcellinida: Hyalospheniidae), a new testate amoeba from Sphagnum peatlands in Bulgaria. Acta Zoologica Bulgarica 70 (2): . 
- Valkanov A (1932) Notizen über das Leben bulgarischer Hochgebirgsseen. Arbeiten der Bulgarischen Naturforschenden Gesellschaft, Sofia 15-16: 207-226. [In Bulgarian, with German summary].

- Valkanov A (1934) Beitrag zur Hydrofauna Bulgariens. Sofia 1-32. [In Bulgarian, with German summary].

\section{Supplementary materials}

\section{Suppl. material 1: Checklist of Sphagum-dwelling testate amoebae from Bulgaria_Distribution data doi}

Authors: Nikola Bankov, Milcho Todorov and Anna Ganeva

Data type: Distribution data

Brief description: Data for the distribution of testate amoebae in Sphagnum mosses in Bulgaria on the basis of literature and of additional data, obtained in our research over the past two years.

Filename: Checklist of Sphagum-dwelling testate amoebae from Bulgaria_Distribution data.xlsx Download file (364.00 kb)

\section{Suppl. material 2: Sampling sites information doi}

Authors: Nikola Bankov, Milcho Todorov and Anna Ganeva

Data type: Sampling sites data

Brief description: Data concerning sampling sites, including date, region, altitude, coordinates, Sphagnum moss species, as well as many physical and chemical parameters of the sampling sites.

Filename: Checklist Sphagnum-dwelling testate amoebae in Bulgaria_Sampling sites data.xlsx Download file (22.89 kb) 